\title{
Extreme Ultraviolet Variability Experiment (EVE) on the Solar Dynamics Observatory (SDO): Overview of Science Objectives, Instrument Design, Data Products, and Model Developments
}

\author{
T.N. Woods · F.G. Eparvier · R. Hock • A.R. Jones · D. Woodraska • D. Judge • \\ L. Didkovsky · J. Lean · J. Mariska · H. Warren · D. McMullin · P. Chamberlin • \\ G. Berthiaume · S. Bailey · T. Fuller-Rowell $\cdot$ J. Sojka $\cdot$ W.K. Tobiska $\cdot$ R. Viereck
}

Received: 5 October 2009 / Accepted: 19 November 2009 / Published online: 12 January 2010

(C) The Author(s) 2010. This article is published with open access at Springerlink.com

The Solar Dynamics Observatory

Guest Editors: W. Dean Pesnell, Phillip C. Chamberlin, and Barbara J. Thompson

T.N. Woods $(\varangle) \cdot$ F.G. Eparvier · R. Hock · A.R. Jones $\cdot$ D. Woodraska

Laboratory for Atmospheric and Space Physics, University of Colorado, 1234 Innovation Dr., Boulder, CO 80303, USA

e-mail: tom.woods@lasp.colorado.edu

D. Judge · L. Didkovsky

Space Sciences Center, University of Southern California, Los Angeles, CA 90089, USA

J. Lean · J. Mariska $\cdot$ H. Warren

Naval Research Laboratory, 4555 Overlook Ave. S.W., Washington, DC 20375, USA

D. McMullin

Space Systems Research Corporation, 1940 Duke St., Alexandria, VA 22314, USA

P. Chamberlin

NASA Goddard Space Flight Center, Greenbelt, MD 20771, USA

G. Berthiaume

Lincoln Laboratory, Massachusetts Institute of Technology, Lexington, MA 02420, USA

S. Bailey

Electrical and Computer Engineering Department, Virginia Tech, Blacksburg, VA 24061, USA

T. Fuller-Rowell

CIRES University of Colorado and NOAA Space Weather Prediction Center, 325 Broadway, Boulder, CO 80305, USA

J. Sojka

Center for Atmospheric and Space Sciences, Utah State University, Logan, UT 84322, USA

W.K. Tobiska

Space Environment Technologies, 1676 Palisades Dr., Pacific Palisades, CA 90272, USA

R. Viereck

NOAA Space Weather Prediction Center, 325 Broadway, Boulder, CO 80305, USA 
Abstract The highly variable solar extreme ultraviolet (EUV) radiation is the major energy input to the Earth's upper atmosphere, strongly impacting the geospace environment, affecting satellite operations, communications, and navigation. The Extreme ultraviolet Variability Experiment (EVE) onboard the NASA Solar Dynamics Observatory (SDO) will measure the solar EUV irradiance from 0.1 to $105 \mathrm{~nm}$ with unprecedented spectral resolution $(0.1 \mathrm{~nm})$, temporal cadence (ten seconds), and accuracy (20\%). EVE includes several irradiance instruments: The Multiple EUV Grating Spectrographs (MEGS)-A is a grazingincidence spectrograph that measures the solar EUV irradiance in the 5 to $37 \mathrm{~nm}$ range with 0.1-nm resolution, and the MEGS-B is a normal-incidence, dual-pass spectrograph that measures the solar EUV irradiance in the 35 to $105 \mathrm{~nm}$ range with $0.1-\mathrm{nm}$ resolution. To provide MEGS in-flight calibration, the EUV SpectroPhotometer (ESP) measures the solar EUV irradiance in broadbands between 0.1 and $39 \mathrm{~nm}$, and a MEGS-Photometer measures the Sun's bright hydrogen emission at $121.6 \mathrm{~nm}$. The EVE data products include a near real-time space-weather product (Level 0C), which provides the solar EUV irradiance in specific bands and also spectra in 0.1-nm intervals with a cadence of one minute and with a time delay of less than 15 minutes. The EVE higher-level products are Level 2 with the solar EUV irradiance at higher time cadence $(0.25$ seconds for photometers and ten seconds for spectrographs) and Level 3 with averages of the solar irradiance over a day and over each one-hour period. The EVE team also plans to advance existing models of solar EUV irradiance and to operationally use the EVE measurements in models of Earth's ionosphere and thermosphere. Improved understanding of the evolution of solar flares and extending the various models to incorporate solar flare events are high priorities for the EVE team.

Keywords EVE $\cdot$ SDO $\cdot$ Solar EUV irradiance $\cdot$ Space weather research

\section{Introduction}

The Solar Dynamics Observatory (SDO) is the first spacecraft in NASA's Living With a Star (LWS) program and is scheduled for a nominal five-year mission starting in February 2010 (or later). The goal of the SDO mission is to understand solar variability and its societal and technological impacts. SDO will investigate how the Sun's magnetic field is generated and structured and how this stored energy is converted and released into the heliosphere and geospace environment through the solar-wind, energetic-particles, and photon output. An underlying theme of SDO is scientific research to enable improved space-weather predictive capabilities, thus transitioning research to operations.

The EUV Variability Experiment (EVE) is one of three instruments onboard SDO. EVE will measure the solar extreme ultraviolet (EUV) and soft X-ray (XUV) spectral irradiance in order to better understand how solar magnetic activity is manifest in the ultraviolet wavelength ranges that drive the terrestrial upper atmosphere. The Helioseismic and Magnetic Imager (HMI) is a vector magnetograph designed to understand magnetic activity, which is the dominant source for solar variability that EVE will measure. HMI also provides high spatial resolution helioseismic data that can provide forecasting capability for the EUV irradiance, both from subsurface magnetic flux and from far-side imaging. The Atmospheric Imaging Assembly (AIA) will make full-disk solar images at multiple wavelengths to link magnetic changes on the surface and interior to those in the solar atmosphere. Most of the AIA wavelengths overlap with EVE, so integration over the solar disk of the AIA images can be compared to EVE irradiance results. The SDO measurements from EVE, HMI, and AIA will facilitate improved understanding of irradiance variations, flares, and coronal 
mass ejections (CMEs), for use in ionosphere and thermosphere models for space-weather operations, to better track satellites, and to manage communication and navigation systems. Furthermore, the EVE observations provide an important extension of the solar EUV irradiance record currently being made by the Solar and Heliospheric Observatory (SOHO) and Thermosphere, Ionosphere, Mesosphere, Energetics and Dynamics (TIMED) spacecraft.

The EVE instrument development coincidently has helped to advance technology in a few areas as briefly noted here. The Charge Coupled Device (CCD) cameras in EVE use radiation-hard, back-illuminated $1024 \times 2048$ CCDs from MIT Lincoln Laboratory and achieve high sensitivity in the EUV range, excellent stability over time, and extremely low noise of about one electron when operated colder than $-70^{\circ} \mathrm{C}$ (Westhoff et al., 2007). The grazing-incidence spectrograph in EVE implements a new, innovative grating design whereby the CCD is near normal incidence, instead of the traditional grazing incidence, so that this spectrograph achieves much higher sensitivity (Crotser et al., 2007). The EUV Spectrophotometer (ESP) instrument in EVE is an expanded version of the SOHO Solar EUV Monitor (SEM) with multiple channels and with a quadrant photodiode that can provide in realtime the location of flares on the solar disk (Didkovsky et al., 2010). As a final technology example, the EVE Solar Aspect Monitor (SAM) channel is a pinhole camera to provide not only alignment information for EVE in visible light but also solar X-ray spectra and images through detecting individual X-ray photon events (Hock et al., 2010).

This paper describes the science objectives, instrumentation, data products, and models for the EVE program. Hock et al. (2010) and Didkovsky et al. (2010) provide more complete instrument description and details of pre-flight calibration results.

\section{EVE Science Plan}

The EUV photons that EVE measures originate in the Sun's chromosphere, transition region, and corona and deposit their energy in the Earth's ionosphere and thermosphere, thus directly connecting the Sun and the Earth in just eight minutes. The solar output in the EUV $(10-121 \mathrm{~nm})$ and XUV $(0.1-10 \mathrm{~nm})$ spectrum varies with solar activity from a factor of two to several orders of magnitude depending on wavelength, and on timescales from seconds and minutes (flares) to days and months (solar rotation) to years and decades (solar sunspot or magnetic cycle) (Woods and Rottman, 2002). The EUV and XUV irradiance is the primary energy input to the Earth's upper atmosphere: heating the thermosphere, creating the ionosphere, and initiating many complex photochemical reactions and dynamical motions. Fluctuating EUV and XUV irradiance drives variability in the atmosphere, affecting satellite operations, navigation systems, and communications. For example, the heating of the thermosphere by solar EUV radiation increases the neutral density with higher levels of solar activity and thus increases satellite drag (Picone, Emmert, and Lean, 2005; Marcos, Bowman, and Sheehan, 2006; Woodraska, Woods, and Eparvier, 2007; Bowman et al., 2008a, 2008b). As shown in Figure 1, the neutral density changes by more than a factor of ten at $500 \mathrm{~km}$ over the 11-year solar cycle, while the thermospheric temperature changes by a factor of 1.5. A small number of previous missions have measured the solar EUV irradiance and produced daily-averaged irradiance spectra (Woods et al., 2004), but none have done so with the accuracy and high time cadence of EVE. EVE will undertake the first comprehensive study of the solar EUV irradiance variability on the time scale of flares. 

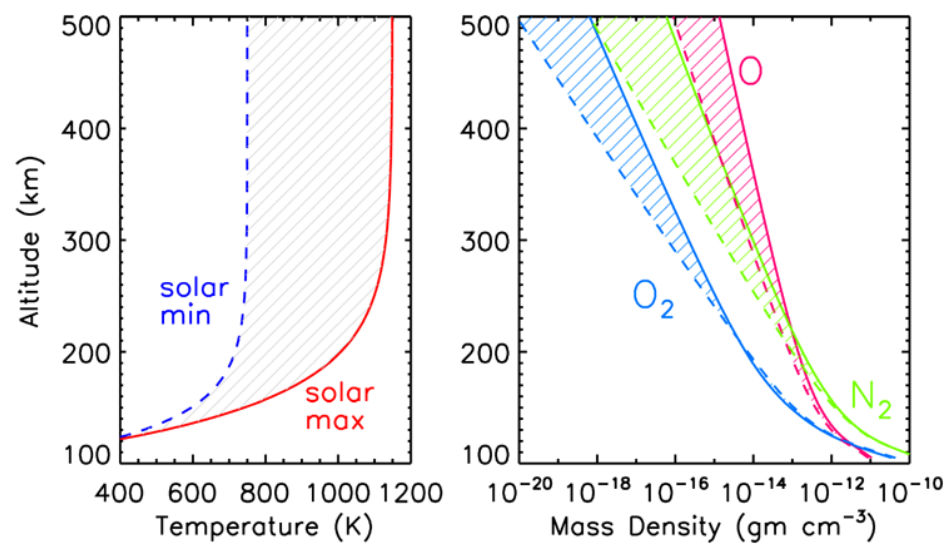

Figure 1 Solar-cycle variation of the thermospheric temperature and neutral density. These are global estimates from the NRLMSIS model, illustrating the large increases in average conditions from solar-cycle minimum to maximum.

\subsection{EVE Science Objectives}

EVE's measurements, modeling activities, and collaborations with the other SDO instruments pursue the following four scientific objectives:

i) specify the solar EUV irradiance and its variability on multiple times scales from seconds to years,

ii) advance current understanding of how and why the solar EUV spectral irradiance varies,

iii) improve the capability to predict (both nowcast and forecast) the EUV spectral variability, and

iv) understand the response of the geospace environment to variations in the solar EUV spectral irradiance and the impact on human endeavors.

\subsubsection{EVE Objective 1-Specify Solar EUV Irradiance}

The first objective of EVE, and its highest priority, is the acquisition of a suitable database to characterize the solar EUV irradiance spectrum and its variations during flares, activeregion evolution, and the solar cycle. Without such observations, the NASA Living With a Star (LWS) program will not be able to develop reliable space environment climatologies. For thermosphere and ionosphere energy-input comparisons, Knipp et al. (2005) demonstrated the dominance of EUV energy versus Joule heating and particle precipitation for Solar Cycles 22 and 23. Figure 2 illustrates modeled variations in the total EUV energy (Lean et al., 2003), compared with that of solar-wind particles, during recent solar cycles.

The solar EUV spectrum (Figure 3 ) is comprised of thousands of emission lines and a few continua whose irradiances span more than four orders of magnitude. This radiation is emitted from the Sun's chromosphere, transition region, and corona at temperatures in the $10^{4}$ to $10^{6} \mathrm{~K}$ range. EUV radiation varies continuously at all wavelengths with hotter emissions generally varying more. Because lines at similar wavelengths can originate from sources on the Sun with different temperatures and densities, the EUV irradiance variability displays complex time-dependent wavelength dependences (see, e.g., Woods and Rottman, 2002). The current TIMED Solar EUV Experiment (SEE) measurements (Woods et al., 2005) have improved the absolute accuracy of the solar EUV spectral irradiances to about $20 \%$, and the 
Figure 2 Key solar-energy input for Earth's ionosphere and thermosphere. Shown are variations in the daily total energy of EUV photons at wavelengths less than $120 \mathrm{~nm}$, compared with the kinetic energy in solar-wind particles (mainly protons). (Updated from Lean, 2005).
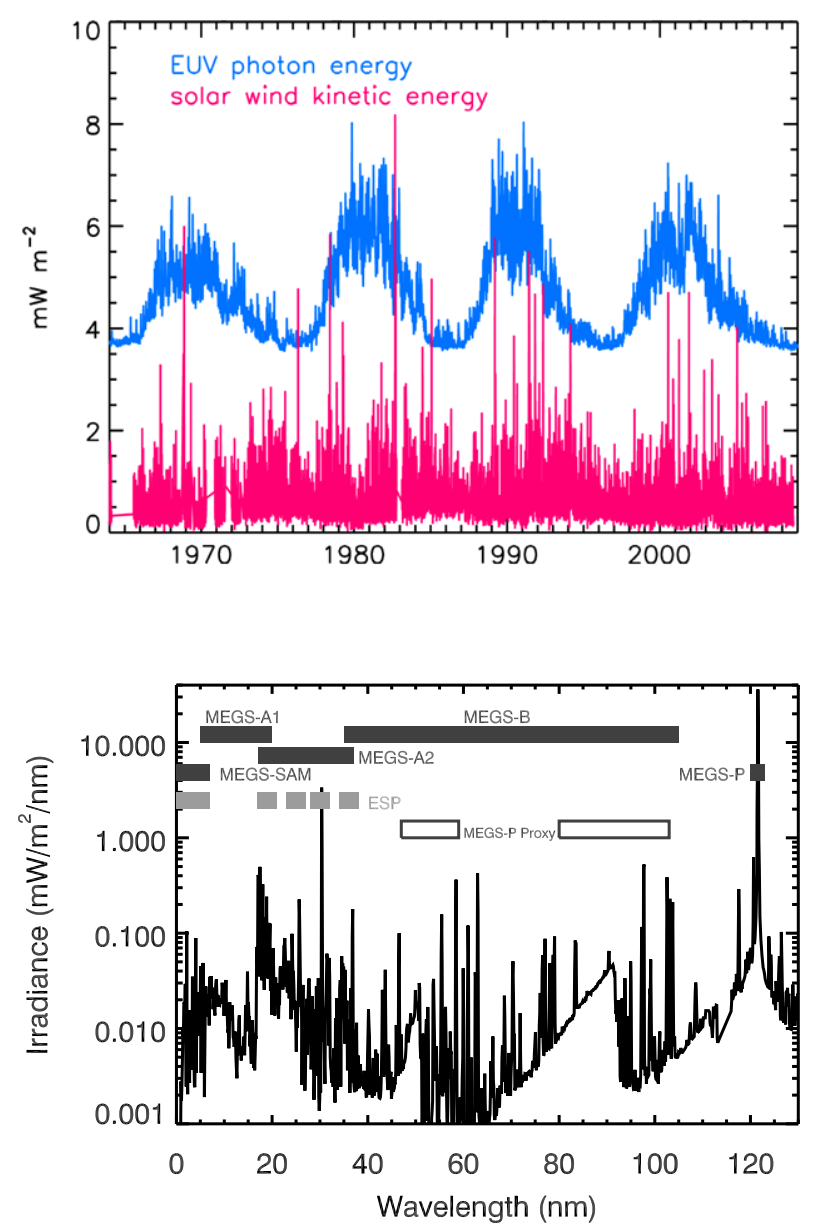

Wavelength $(\mathrm{nm})$
Figure 3 EVE instrument spectral ranges. The wavelength coverage of the various EVE instruments plotted over the solar minimum spectrum obtained with the prototype EVE instrument on 14 April 2008 (Chamberlin et al., 2009).

EVE measurements will further increase this accuracy at some wavelengths to about $10 \%$ over the SDO mission and with much higher spectral resolution of $0.1 \mathrm{~nm}$. The TIMED/SEE measurements have also achieved the first systematic characterization of the EUV spectral irradiance variations during solar rotation and the descending phase of a solar cycle, as shown in Figure 4 for SEE Level 3 product (Version 10) in 1-nm intervals. EVE will expand these observations to longer time scales, a factor of ten higher spectral resolution and much higher temporal cadence.

The historical solar EUV irradiance database, including the TIMED/SEE measurements, consists mainly of daily measurements, albeit with many large gaps such as from 1979 to 2002. Observations of the solar EUV irradiance with higher temporal cadence, as needed to study flares, are severely limited. The SOHO Solar EUV Monitor (SEM, Judge et al., 1998) and GOES/XRS (Garcia, 1994) measurements do provide high time cadence but only in a few broad wavelength bands. EVE's spectrally-resolved observations with a temporal cadence of ten seconds will greatly advance the specification and understanding of the spectral variations during flare events throughout the XUV and EUV spectrum. 
Figure 4 Solar EUV irradiance variations of the 11-year solar cycle. The TIMED/SEE results (line) agree very well with the UARS results (diamonds) in the far ultraviolet range (Woods et al., 2005).

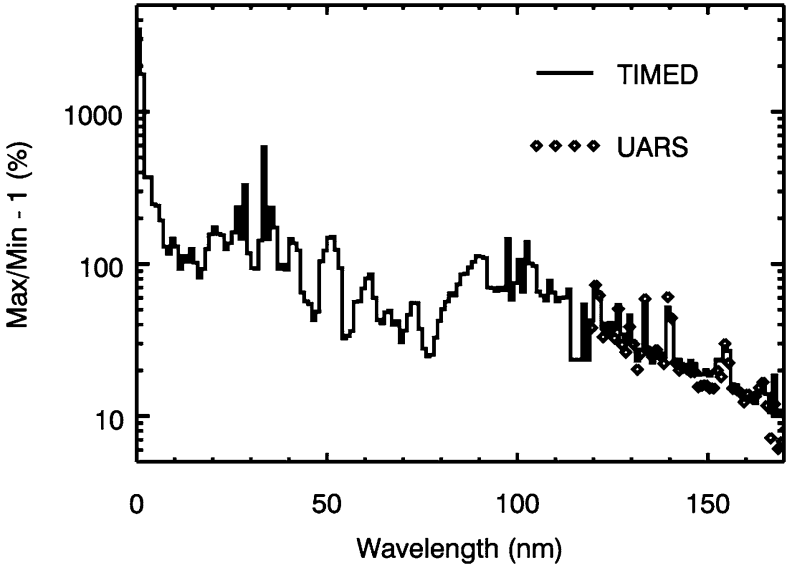

\subsubsection{EVE Objective 2 - Understand Why Solar EUV Irradiance Varies}

Extensive, multi-faceted investigations are planned to advance understanding of the sources of EUV irradiance variations within a physical framework. This understanding is needed to develop a predictive capability for past and future space-environment climatologies, and for verifying the direct EUV irradiance observations. Models will be developed to account for the observed EUV irradiances and their variations, with traceability to magnetic-flux emergence and the solar dynamo. This approach will be accomplished in collaboration with complementary SDO efforts, including those of the HMI and AIA instruments and other space missions. Auxiliary data from space and from the ground, e.g. by the Synoptic Optical Long-term Investigations of the Sun (SOLIS), Big Bear Solar Observatory programs, and the National Science Foundation's Precision Solar Photometric Telescope (PSPT), will also be used.

EUV radiation emerges from the outer layers of the Sun's atmosphere, which are sufficiently hot to excite highly-ionized species of gases; however, the solar atmosphere is not homogeneous, as the images in Figures 5 and 6 clearly illustrate. The EUV brightness inhomogeneities on the Sun are regions where magnetic fields alter the solar atmosphere. The fundamental determinant of the EUV spectrum is therefore the solar dynamo. There is a broad conceptual appreciation of the connections that relate the solar dynamo to surface magneticflux emergence, upward field propagation and expansion, active-region and coronal-hole formation, and EUV irradiance modulation, but the physical links remain largely undetermined. A self-consistent, end-to-end formulation that quantitatively relates the net EUV radiation to subsurface magnetic fields does not yet exist.

The recent approach of using differential-emission measures to model solar EUV irradiance variations (Warren, Mariska, and Lean, 2001) is a first step to quantify crucial physical processes. Emission-measure distributions are derived from spatially and spectrally resolved measurements of line intensities, and describe the temperature and density structure of the solar atmosphere. A quantitative estimate of the EUV radiation spectrum is possible by combining intensities calculated from emission-measure distributions of representative EUV sources, determinations from full-disk images (such as in Figures 5 and 6) of the fractional coverage of these sources, limb brightening, atomic properties and abundances. The EVE investigation will utilize the AIA full-disk images to quantify and track the fractional occurrence of EUV irradiance sources (e.g. active regions, active network, coronal holes) 

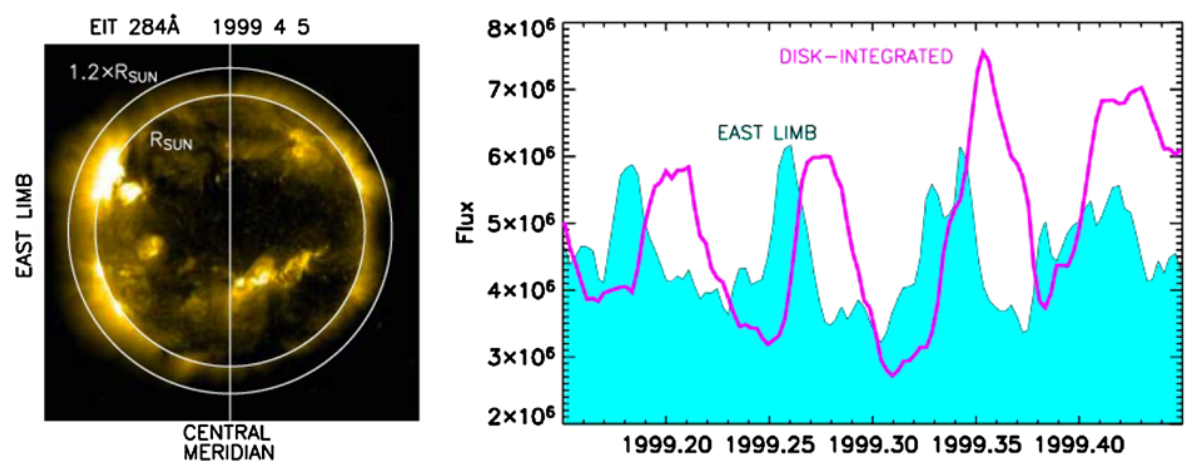

Figure 5 Forecasting technique for solar irradiance. Shown on the left are aspects of the solar disk utilized to develop a predictive capability. On the right, two time series derived from two different non-intersecting subsets of the EIT $28.4 \mathrm{~nm}$ images are compared. The east-limb flux (shaded region) is the signal from only those pixels between 1 and $1.2 \times R_{\text {Sun }}$ in the eastern half of the image. The disk-integrated flux (solid line) is the signal from all pixels on the disk. This forecast technique is described in more detail by Lean, Picone, and Emmert (2009).
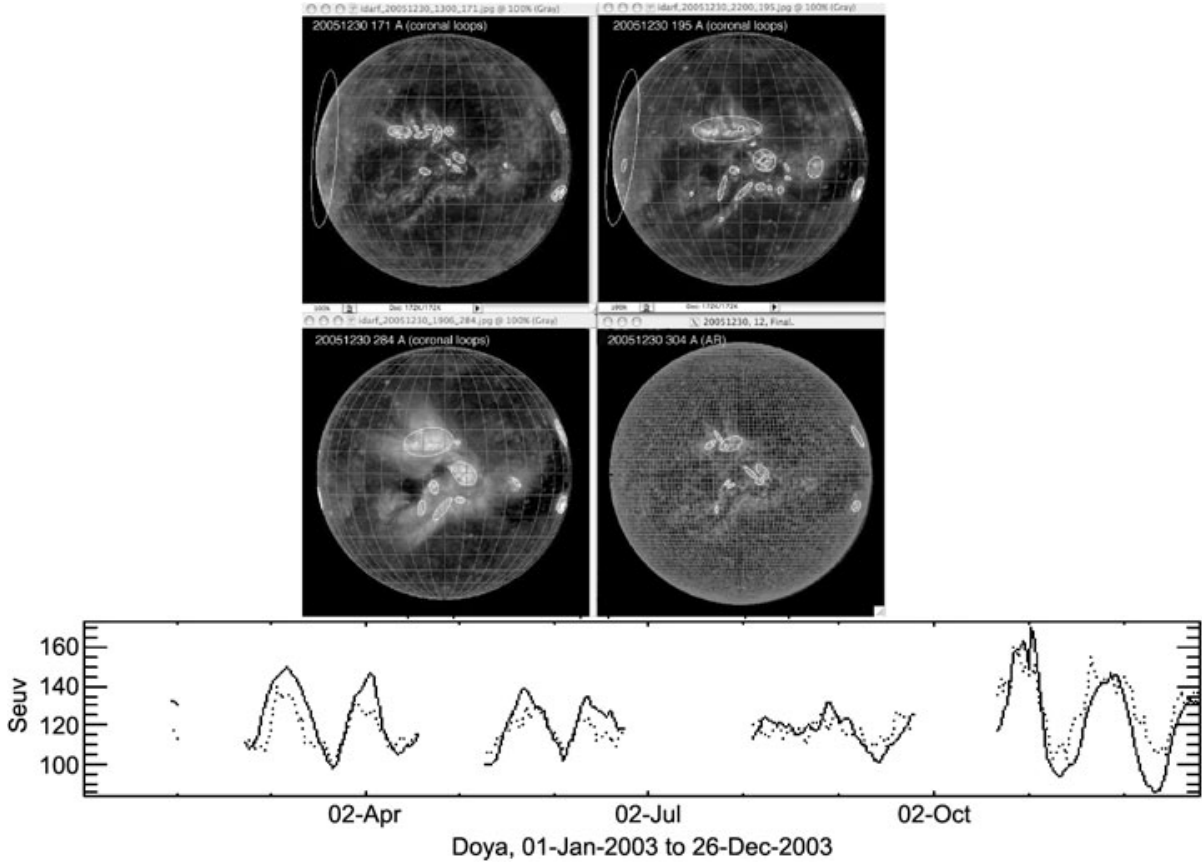

Figure 6 SOHO images are processed by the SET IDAR algorithm to extract active regions and coronal loops for three to seven days operational solar-irradiance forecasting. The $30.4 \mathrm{~nm}$ active-region and plage data are extracted to forecast the one-day prediction of the F10.7 index (SEUV, dotted line) for use in the JB2006 and JB2008 models. The actual F10.7 index is shown as the solid line.

throughout the SDO mission. Comparisons of the EUV irradiance modeled from AIA observations with the direct EVE measurements will reveal how well, or how poorly, this physical theory accounts for irradiance variations. 
With quantitative relationships between magnetic structures in the solar images and the measured EUV irradiance established, subsequent exploration of the physical connections between EUV irradiance and surface magnetic fields becomes possible. SDO's HMI (and also ground-based magnetograms) map surface magnetic fields. Combining the HMI and AIA images (made at different temperatures) provides empirical tracing of magnetic fields from their relatively compact photospheric footprints to the more expanded EUV sources. Comparisons with modeled extrapolations of surface fields will help characterize the relationship between the fields and EUV brightness. Initial simulations have elucidated the primary role of footprint field strength and the less important role of loop length in accounting for observed coronal irradiance changes during solar rotation and the solar cycle. Analogous studies will combine data and results from the EVE instruments and other SDO instruments to quantify the relationship between surface magnetic structures and the EUV irradiance.

Once the coronal and chromospheric magnetic-field configurations that cause bright and dark EUV sources are properly connected to surface magnetic fields, the EUV irradiance variations become amenable to study in terms of source emergence, meridional transport and diffusion, as encapsulated in current flux-transport models (Wang, Lean, and Sheeley, 2000; Wang, Sheeley, and Lean, 2000). For example, the NRL flux transport model (Wang and Sheeley, 1991) may be used to simulate the evolution of the large-scale surface magnetic field for comparison with synoptic changes in the chromosphere and corona deduced from the AIA images. Relationships between subsurface signatures of flux emergence derived from HMI images, and their surface manifestations ultimately link the solar dynamo and the EUV radiation. New understanding of the solar dynamo and its possible long-term evolution and intermittent behavior (such as during and since the Maunder Minimum) will then be directly applicable to studying the EUV irradiance variations on longer time scales, beyond those accessible to space-based observations (see, e.g., Wang, Lean, and Sheeley, 2005).

Section 5 describes in more detail the plans to update the physics-based NRLEUV model (Warren, Mariska, and Lean, 2001), the empirical Flare Irradiance Spectral Model (FISM) (Chamberlin, Woods, and Eparvier, 2007, 2008), and the hybrid Solar Irradiance Platform (SIP) system (Tobiska, 2004, 2008; Tobiska and Bouwer, 2006; Tobiska et al., 2000). Physics-based models provide more insight into the causes of the solar variability and better links to the solar image results. Empirical models that relate variability of proxies to the solar variability at other wavelengths can provide accurate and easy-to-calculate results for space-weather research and operations when direct measurements are not available. Hybrid systems, such as SIP, combine real-time data, reference spectra, empirical, and physics-based models to report, specify, and forecast the solar irradiances.

\subsubsection{EVE Objective 3 - Forecast Solar EUV Variations}

The application of the physical understanding and specification of the solar EUV irradiance developed in EVE Objectives 1 and 2 will facilitate a unique, and hitherto unavailable, capability for EUV irradiance predictions on multiple time scales associated with the solar cycle, rotation, and flares. Predictions for the various time scales ranging from the long-term solar cycle to the short-term flare eruptions require different techniques.

Solar-activity cycles are currently predicted in terms of sunspot numbers or $10.7 \mathrm{~cm}$ radio fluxes. NASA uses such predictions for planning shuttle and space station activities, for example. Most previous predictions use either statistical regression techniques based on average properties and mean behavior of sunspot patterns or the geophysical precursor technique, which recognizes the extended nature of the solar cycle (Hathaway, Wilson, and Reichmann, 1999). Were the EUV irradiance and its variability better known, these predictions could be transformed empirically to much improved equivalent EUV energies. EVE 
measurements, with high accuracy and repeatability, will greatly improve the specification of the relationship between EUV irradiance and sunspot numbers (or $10.7 \mathrm{~cm}$ fluxes) to facilitate useful nowcasts and forecasts of present and future long-term irradiance cycles.

Predicting the details of day-to-day EUV irradiance variations associated with solar rotations requires a different approach. The US Air Force has an operational requirement for predicting space weather on a three-hour time granularity to 72 hours and a daily-forecast requirement to seven days or beyond while NOAA provides public predictions of radio flux to 45 days. Commercial aerospace organizations use commercially provided irradiance predictions out to five solar rotations. Because solar EUV radiation is a primary driver of geospace fluctuations on these time scales, the predictions ultimately require reliable forecasts of future solar EUV irradiance. A new capability will be developed to predict day-to-day EUV irradiances by using HMI far-side images and AIA limb emission to quantify emerging flux and active regions soon to rotate onto the face of the Sun visible at the Earth. The physical associations between surface fields and active regions in the chromosphere and corona (developed in EVE Objective 2) then permit quantitative forecasts of EUV irradiance using the far-side and east-limb active regions. The far-side active regions are currently predicted using helioseismology with SOHO/MDI data (Lindsey and Braun, 2000) and backscattered H I Lyman- $\alpha$ emissions with SOHO/SWAN data (Bertaux et al., 2000). Fontenla et al. (2009) provide examples of forecasting the solar EUV irradiance using these far-side techniques.

As Figures 5 and 6 demonstrate, the emission on the east limb of the solar disk is a useful predictor of full-disk flux (irradiance) from three to nine days later. Separately, EVE's direct observations acquired in Objective 1 provide a database to quantify and validate this association using AIA images, and to develop, as well, an independent statistical predictive capability from accumulated characterizations of EUV irradiance variations at particular phases of the solar cycle. Together, the complementary physical and statistical approaches enable state-of-the-art near-term EUV irradiance forecasts. The database of ten-second EUV irradiance spectra will be particularly useful for nowcasting space weather.

The ability to forecast short-term solar eruptions has progressed in the past few years as a result of empirical associations between certain magnetic-field configurations (such as helicity features, dimmings, and shadows) and subsequent flares and coronal mass ejections. With its high time cadence, EVE will record the increase in EUV irradiance that accompanies each and every flare throughout the SDO mission. This unique database will facilitate classifications of the types and nature of geoeffective flares, ones that have sufficient brightness to dominate the disk-integrated signal during an eruptive event. This database will enable empirical associations with flare precursors detected in HMI and AIA images. EVE will develop, in collaboration with the SDO imaging teams, physical descriptions of the associations of precursor magnetic field configurations and subsequent EUV irradiance enhancements. These studies will contribute to more reliable short-term forecasts of EUV irradiance levels and hence of abrupt space-weather phenomena.

\subsubsection{EVE Objective 4 - Understand Response of Geospace Environment}

EVE will provide reliable knowledge of the solar EUV spectrum and its variability that the geophysics community has sought for decades, and without which LWS cannot fully succeed. Variations in EUV irradiance initiate space-weather phenomena through both direct and indirect processes and are, consequently, crucial inputs for many geospace models. The direct terrestrial effects of solar EUV electromagnetic radiation are well recognized (National Space Weather Plan, 2000). The EUV irradiances specified by EVE will enable progress in understanding, specifying, and forecasting myriad space-weather phenomena, including spacecraft drag, communications, and navigation. 
Through both direct and collaborative methods, the EVE team will support modeling and interpretation of the neutral atmosphere and ionosphere responses to EUV irradiance variations. Examples of such studies include simulations using the NOAA three-dimensional Coupled Thermosphere Ionosphere Plasmasphere electrodynamics model (CTIPe; FullerRowell et al., 1996) and the Utah State University Time-Dependent Ionospheric Model (TDIM; Sojka, 1989; Schunk et al., 2004) with a hierarchy of inputs ranging from direct high time-cadence observations of flares, modeled flare spectra, active-region time scale and near-term forecast responses, solar-cycle-induced variations and extreme conditions of solar activity. EVE will enable the solar component in the day-to-day variability of ionospheric electron density and total electron content to be quantified for the first time. Such a comprehensive study of atmosphere-ionosphere responses to EUV radiation has never been conducted. Prior studies, undertaken only infrequently because of their interdisciplinary nature, have suffered from the quality of their adopted EUV irradiance inputs. Only very recently, for example, were sufficient interdisciplinary resources brought together to simulate the ionospheric response to EUV spectrum changes during a flare (see, e.g., Pawlowski and Ridley, 2008; Smithtro and Solomon, 2008).

\subsection{EVE Science Team}

The EVE Principal Investigator is Tom Woods at the University of Colorado's Laboratory for Atmospheric and Space Physics (LASP). The EVE scientists include Frank Eparvier and Andrew Jones at LASP, Darrell Judge and Leonid Didkovsky at the University of Southern California (USC), Judith Lean, Don McMullin, John Mariska, and Harry Warren at the Naval Research Laboratory (NRL), Greg Berthiaume at the Massachusetts Institute of Technology Lincoln Laboratory (MIT-LL), and Scott Bailey at Virginia Tech.

LASP provides overall EVE project management, instrument design, fabrication, calibration, instrument operations, and data processing software development. USC contributes a portion of the flight hardware and expertise in solar EUV irradiance measurements. MIT leads the development of CCD detectors (Westhoff et al., 2007). NRL undertakes solar spectral-irradiance modeling, in particular improvements to the NRLEUV semi-empirical irradiance variability model (Warren, Mariska, and Lean, 2001; Lean et al., 2003).

The EVE Science Team also includes several collaborators whose participation is vital to EVE's success: Tim Fuller-Rowell and Rodney Viereck (NOAA Space Weather Prediction Center, SWPC), Jan Sojka (Utah State University, USU), and Kent Tobiska (Space Environment Technologies, SET) participate in various aspects of the space-weather and operations effort through geospace and solar operational modeling. They will assist in transitioning EVE research to operations. In particular, EVE data will be used to improve, validate, or constrain solar irradiance models, such as SIP (Tobiska, 2004, 2008; Tobiska and Bouwer, 2006; Tobiska et al., 2000), and atmospheric models, such as CTIPe (Fuller-Rowell et al., 1996), Global Assimilation of Ionospheric Measurements (GAIM; Schunk et al., 2004), NRLMSIS (Picone et al., 2002), JB2006 (Bowman et al., 2008a), JB2008 (Bowman et al., 2008b), and a newly developed improved density specification model that takes into account an extensive density database and includes direct formulation with the Mg index (Emmert, Picone, and Meier, 2008). These atmospheric models are described in more detail in Section 6.

\subsection{EVE Measurement Requirements}

To help meet the EVE objectives, the EVE instrument suite will measure the spectral irradiance from 0.1 to $5 \mathrm{~nm}$ at $1-\mathrm{nm}$ resolution, from 5 to $105 \mathrm{~nm}$ with a resolution of $0.1 \mathrm{~nm}$, plus 
the hydrogen Lyman- $\alpha$ line at $121.6 \mathrm{~nm}$ with 1-nm resolution. The full spectral range will be measured every ten seconds, continuously (except during satellite eclipse periods and planned calibration activities). The absolute accuracy of EVE's spectral irradiance measurements will be better than $25 \%$ throughout the nominal five-year mission. The pre-flight tests and calibrations verify that EVE meets these requirements, and at many wavelengths EVE greatly exceeds the $25 \%$ accuracy. The measurement requirements represent significant improvements over previous measurements, especially noteworthy for the spectral resolution, time cadence, and duty cycle.

\section{EVE Instrumentation}

To meet the measurement and accuracy requirements, the EVE suite is composed of several instruments as listed in Table 1. The wavelength coverage of all instruments is shown in Figure 3, along with the solar-cycle minimum spectrum obtained with the prototype EVE onboard a sounding rocket flight on 14 April 2008 (Chamberlin et al., 2009). Eparvier et al. (2004), Crotser et al. (2004, 2007), Chamberlin et al. (2007), Didkovsky et al. (2007, 2010), and Hock et al. (2010) provide details of the optical designs for the EVE instruments, but a brief overview of the instruments is included here.

The primary, high-spectral-resolution irradiance measurements are made by the Multiple EUV Grating Spectrographs (MEGS) (Crotser et al., 2004, 2007), which have heritage from the TIMED/SEE EUV Grating Spectrograph (EGS) (Woods et al., 2005). The MEGS is composed of two spectrographs: MEGS-A is a grazing-incidence spectrograph covering the 5 to $37 \mathrm{~nm}$ range, and MEGS-B is a two-grating, cross-dispersing spectrograph covering the 35 to $105 \mathrm{~nm}$ range. Both MEGS-A and B have 0.1-nm spectral resolution. Included as part of the MEGS-A package is a pinhole camera for use as a solar aspect monitor (MEGSSAM) to provide a pointing reference for the EVE instruments. MEGS-SAM will also make a spectral measurement of the solar irradiance in the 0.1 to $5 \mathrm{~nm}$ wavelength range at approximately 1-nm resolution. In addition, a photodiode with a filter to isolate Lyman- $\alpha$ at $121.6 \mathrm{~nm}$ (MEGS-P) is part of the MEGS-B. This measurement is used to track potential changes in the sensitivity of the MEGS on timescales of weeks and months. Annual sounding rocket underflights of similar instruments will track longer-term changes in the sensitivity of the EVE instruments. Eparvier et al. (2004), Crotser et al. (2004, 2007), Chamberlin et al. (2007), and Hock et al. (2010) provide more details about LASP's MEGS and its calibration results.

Additional onboard short-term calibration tracking is achieved by redundant, lowerspectral-resolution measurements at select bandpasses, made by the EUV Spectrophotometer (ESP). The ESP is a transmission grating and photodiode instrument similar to the SOHO/SEM (Judge et al., 1998). ESP has four channels centered on 18.2, 25.7, 30.4, and $36.6 \mathrm{~nm}$ that are each approximately $4 \mathrm{~nm}$ in spectral width. The ESP also has a central, zeroth-order diode with a filter to make the primary irradiance measurement in the 0.1 to $7 \mathrm{~nm}$ range. The ESP measurements are made at a high time cadence ( 0.25 seconds) and so are useful as quick indicators of space-weather events such as flares. Didkovsky et al. (2007, 2010) provide more details about USC's ESP and its calibration results.

\subsection{MEGS-A Instrument}

The MEGS-A instrument is an $80^{\circ}$ grazing-incidence, off-Rowland circle spectrograph with a CCD detector to measure the solar spectrum between 5 and $37 \mathrm{~nm}$ at a resolution just 


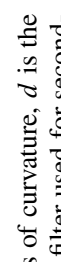

荧

of

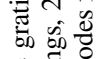

을 은

a 50

位

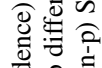

要

은

主

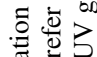

颉口

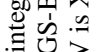

产是

证势

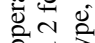

굴

을

50

要

क 5

ব

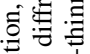

응

s

⿶)

政

要

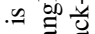

子๐

它 :

苛高

总.

证:

의

要

$\exists$ ช

ㅎํㅇำ

II

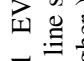

- 00.

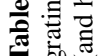

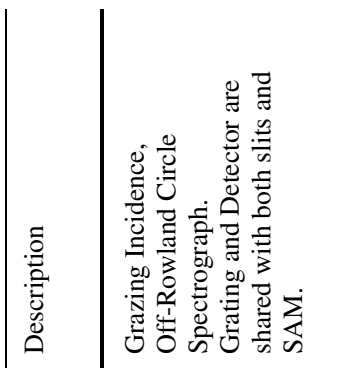

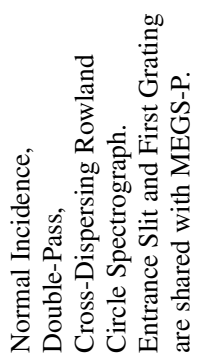

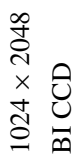

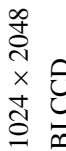

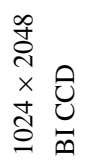

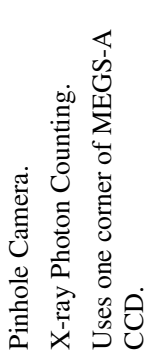

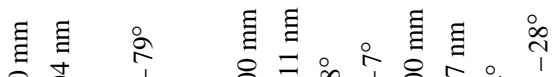

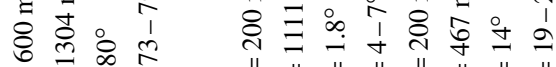

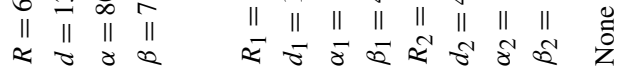

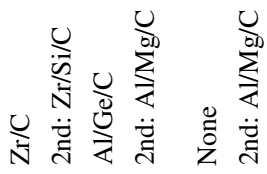

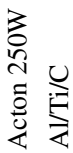

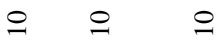

응

ব

૪茞

$\overrightarrow{0} \quad \overrightarrow{0}$

$\infty-$

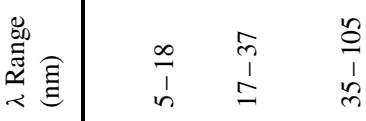

กิ

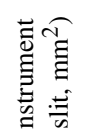

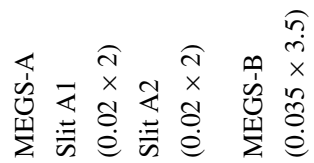

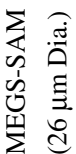




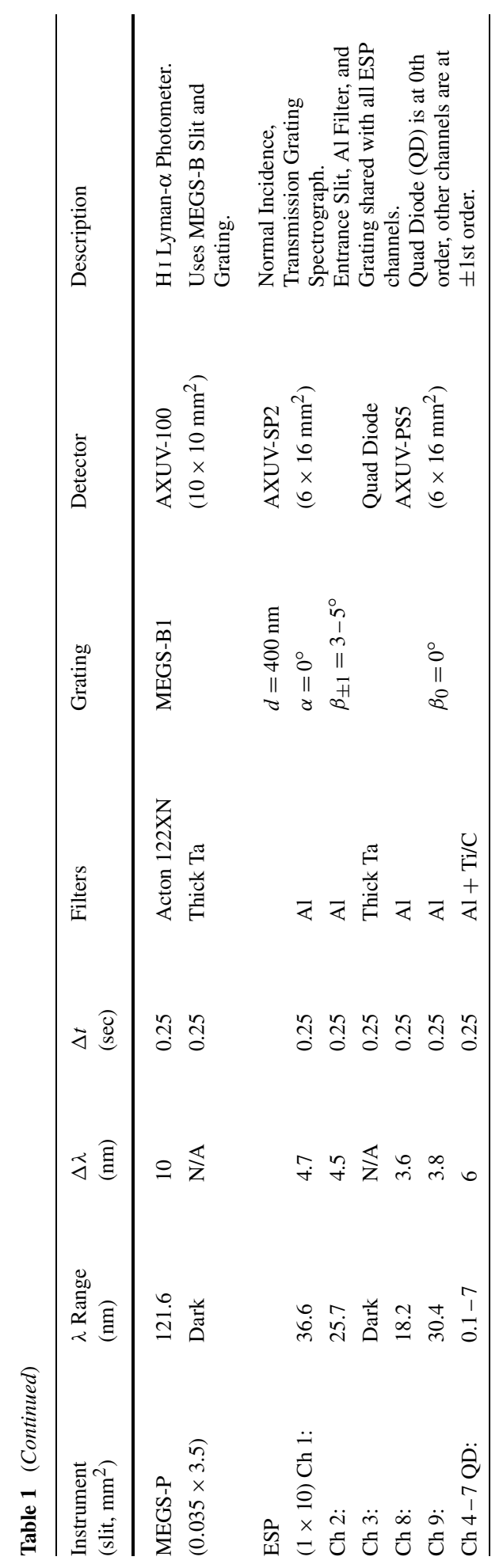


less than $0.1 \mathrm{~nm}$. MEGS-A has two entrance slits, each 20 microns wide and $2 \mathrm{~mm}$ high, oriented top-to-bottom. The off-Rowland circle design is an innovative approach to having the CCD at near-normal incidence, instead of grazing incidence, so that MEGS-A has much higher sensitivity (Crotser et al., 2007). In front of the slits is a filter-wheel mechanism with bandpass-limiting thin foil filters (made by Luxel Corp.). The primary science filters are $\mathrm{Zr}$ $(280 \mathrm{~nm}) / \mathrm{C}(20 \mathrm{~nm})$ for slit 1 to isolate 5 to $18 \mathrm{~nm}$ and $\mathrm{Al}(200 \mathrm{~nm}) / \mathrm{Ge}(20 \mathrm{~nm}) / \mathrm{C}$ $(20 \mathrm{~nm})$ for slit 2 to isolate 17 to $37 \mathrm{~nm}$. Secondary filters are available to further limit the bandpasses of each slit to provide an occasional check on higher orders: $\mathrm{Zr}(230 \mathrm{~nm}) / \mathrm{Si}$ $(120 \mathrm{~nm}) / \mathrm{C}(20 \mathrm{~nm})$ for slit 1 to pass 13 to $18 \mathrm{~nm}$, and $\mathrm{Al}(180 \mathrm{~nm}) / \mathrm{Mg}(300 \mathrm{~nm})$ to pass 25 to $37 \mathrm{~nm}$ for slit 2. The filter-wheel mechanism also has a blanked-off position for dark measurements. The grating, produced by Jobin Yvon (JY), is a spherical holographic

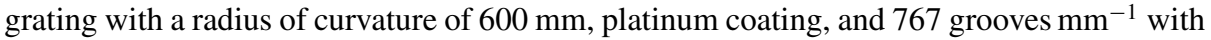
a laminar groove profile to suppress even orders.

The detector for MEGS-A is a back-thinned, back-illuminated, split-frame transfer CCD with $1024 \times 2048$ pixels, built by MIT-LL (Westhoff et al., 2007). The pixel size is $15 \mu \mathrm{m} \times$ $15 \mu \mathrm{m}$. The top and bottom halves, each $512 \times 2048$ pixels, are read out through separate, redundant signal chains (charge amps) in about eight seconds. The gain on the CCD signal is about two electrons per data number (DN), and its noise is about two electrons once its temperature is below about $-70^{\circ} \mathrm{C}$. The $\mathrm{CCD}$ is maintained at $-95^{\circ} \mathrm{C} \pm 5^{\circ} \mathrm{C}$ during flight to suppress noise and to minimize radiation effects in the geosynchronous environment. Furthermore, the CCDs are heavily shielded by about $25 \mathrm{~mm}$ Al to reduce the radiation dose to less than $10 \mathrm{kRad}$ over the SDO mission.

\subsection{MEGS-B Instrument}

The MEGS-B instrument is a normal-incidence, double-pass, cross-dispersing Rowland circle spectrograph with a CCD detector to measure the solar spectrum between 35 to $105 \mathrm{~nm}$ at a resolution just less than $0.1 \mathrm{~nm}$. MEGS-B has a single entrance slit, 35 microns wide and $3.5 \mathrm{~mm}$ high. There are no known reliable bandpass filters for the MEGS-B wavelength range, so a two-grating design isolates the entire 35 to $105 \mathrm{~nm}$ range. A filter-wheel mechanism is also included with clear positions for primary science measurements, a $\mathrm{Al}$ (180 nm) / $\mathrm{Mg}(300 \mathrm{~nm})$ foil filter for higher-order checks, and a blanked-off position for dark measurements. Both MEGS-B gratings are also produced by JY and are spherical holographic gratings with platinum coating and laminar groove profiles to suppress even orders. The first grating has 900 grooves $\mathrm{mm}^{-1}$ and the second has 2140 grooves $\mathrm{mm}^{-1}$. The detector for MEGS-B is identical to the MEGS-A detector and permits about three pixels across the optical resolution of $0.1 \mathrm{~nm}$ and over 100 pixels along the slit image.

\subsection{MEGS-SAM Instrument}

The MEGS-SAM instrument is a pinhole camera within the MEGS-A housing, using a separate aperture, but producing an image of the Sun on a portion of the MEGS-A CCD where the bandpass filter for slit 2 blocks essentially all light. The SAM aperture has a separate filter-wheel mechanism that allows for three modes. In aspect-monitor mode, a UV filter is in place and the resultant image of the Sun is centroided to give pointing information for all of EVE relative to the boresights established during pre-flight calibrations to roughly one arcminute accuracy. In XUV photon-counting mode, a Ti $(300 \mathrm{~nm}) / \mathrm{Al}(150 \mathrm{~nm}) / \mathrm{C}$ $(40 \mathrm{~nm})$ foil filter is in place to isolate 0.1 to $7 \mathrm{~nm}$. This filter is designed to be the same as the ESP $\mathrm{Al}$ and Ti/C filter combination used with ESP's zeroth-order photometer. The pinhole 
and filter are optimized so that single photon events can occur during the ten-second CCD integration. The energy (or wavelength) is determined from the magnitude of each photon event, thus binning photon events from over the entire image of the Sun gives a low $(\approx 1 \mathrm{~nm})$ spectral resolution for the SAM XUV bandpass. Summing consecutive integrations over a few minutes generates XUV images of the Sun. The third mode for SAM has the filter wheel in a blanked-off position for dark measurements. The SAM is normally in its XUV mode.

\subsection{MEGS-P Instrument}

The MEGS-P instrument is an International Radiation Detectors (IRD) silicon photodiode placed at the minus first order of the first MEGS-B grating. In front of the diode is an Acton interference filter to isolate the solar hydrogen Lyman- $\alpha$ line at $121.6 \mathrm{~nm}$. The filter has a bandwidth of $10 \mathrm{~nm}$, but the solar spectrum is such that greater than $99 \%$ of the signal is due to Lyman- $\alpha$. Next to the primary MEGS-P diode is an identical diode that is masked to give simultaneous dark information, used to correct the MEGS-P measurements for background noise induced by particle radiation.

\subsection{ESP Instrument}

The ESP instrument is a non-focusing, broadband spectrograph with a transmission grating and IRD silicon photodiodes similar to the SOHO/SEM instrument (Judge et al., 1998). In front of the entrance slit is an $\mathrm{Al}(150 \mathrm{~nm})$ foil filter made by Luxel Corp. to limit the out-of-band light that enters the instrument. The free-standing transmission grating, made by X-Opt. Inc., is essentially a set of thin bars with no substrate, spaced so that there are 2500 lines $\mathrm{mm}^{-1}$. Silicon photodiodes are placed at both plus and minus first orders and positioned so that the bandpass centers are at 18.2, 25.7, 30.4, and $36.6 \mathrm{~nm}$. Masks in front of the diodes are sized to give approximately 4-nm bandpasses centered on each of these wavelengths. The central, zeroth-order position has a silicon quadrant photodiode with an additional thin foil $\mathrm{Ti}(300 \mathrm{~nm}) / \mathrm{C}(40 \mathrm{~nm})$ filter to isolate 0.1 to $7 \mathrm{~nm}$. The sum of the quadrants gives the solar irradiance in this bandpass. Differencing the quadrants allows for determination of the pointing of the ESP and can also provide the location of flares on the solar disk (Didkovsky et al., 2010). The ESP has a filter-wheel mechanism with Al foil filters for solar measurements, a blank position for dark measurements, and a fused-silica window to check for visible stray light. The ESP has the fastest measurement cadence of all of the instruments in the EVE suite at 0.25 seconds.

\subsection{Other EVE Subsystems}

The EVE instruments are packaged together onto the EVE Optical Package (EOP) as shown in Figure 7. The EOP is mounted to the SDO spacecraft deck using three Ti-flex structures. The microprocessor, interface electronics, control electronics, and most of the power conditioning and regulation electronics are housed in the EVE Electronics Box (EEB). The EEB resides behind the EOP and directly mounts to the SDO spacecraft deck. The interfaces to the SDO spacecraft include unregulated $28 \mathrm{~V} \mathrm{DC}$ for power, 1553 for commands and housekeeping telemetry, and High Speed Bus (HSB) for science telemetry. Several radiators are also part of the EVE package, to passively remove heat by radiating to deep space. The resources for EVE include mass of $54 \mathrm{~kg}$, orbit-average power of $44 \mathrm{~W}$, housekeeping telemetry of two kilobits per second (kbps), and science telemetry of seven Megabits $\mathrm{sec}^{-1}$ (Mbps). 


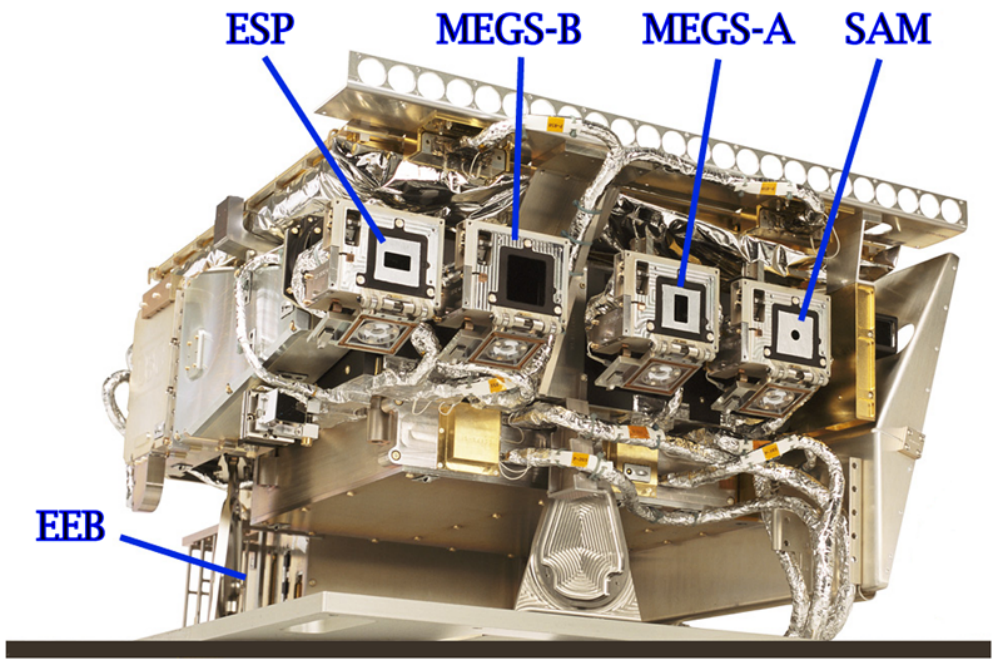

Figure 7 The EVE instruments are mounted onto the EVE Optical Package (EOP), and the EVE Electronics Box (EEB) provides the electrical interfaces to the SDO spacecraft. The entrance baffle in the door mechanisms are indicated for the various MEGS and ESP instruments.

\section{EVE Data Products}

The primary EVE data products are solar EUV irradiances at 0.1-nm and 1-nm resolution at a ten-second time cadence and as daily averages. In addition, specific solar emission lines and broadband irradiances will be extracted and provided at both time cadences. These data products will be available within a day or so of receipt on the ground. Near real-time data products for space-weather operations will also be produced and available within approximately fifteen minutes of ground receipt. The space-weather products will not be as fully corrected or processed as the primary data products, and may consist of solar indices and spectra with preliminary calibration. The EVE space-weather products are intended for use in near real-time applications, such as in ionospheric and thermospheric models and for "nowcasting" short-time-scale solar events such as flares. The following sections describe the EVE data-processing system and data products (Table 2). The raw telemetry records are called Level 0A, and raw data sorted by channel are called Level 0B; neither of these products is discussed below. The algorithms for converting the raw data into irradiance are provided in the instrument calibration papers by Hock et al. (2010) for MEGS and Didkovsky et al. (2010) for ESP.

\subsection{EVE Level 0C - Space Weather Product}

The EVE space-weather products, called Level 0C and Level 0CS, provide the solar EUV irradiance as soon as possible after the observation. To reduce the processing time, only simple forms of the calibration parameters are applied. The solar irradiance is provided as spectra from 6 to $105 \mathrm{~nm}$ in 0.1-nm intervals and for several different spectral bands, each with one-minute cadence and with less than 15-minute latency. The spacecraft and ground systems impose a 15-minute latency, which is adequate for using ionosphere and thermosphere models for space-weather operations (see Section 6). Some of the bands are also provided with about one-minute latency for the most time-critical space-weather operations, namely flare events and predictions for Solar Energetic Particles (SEPs). 


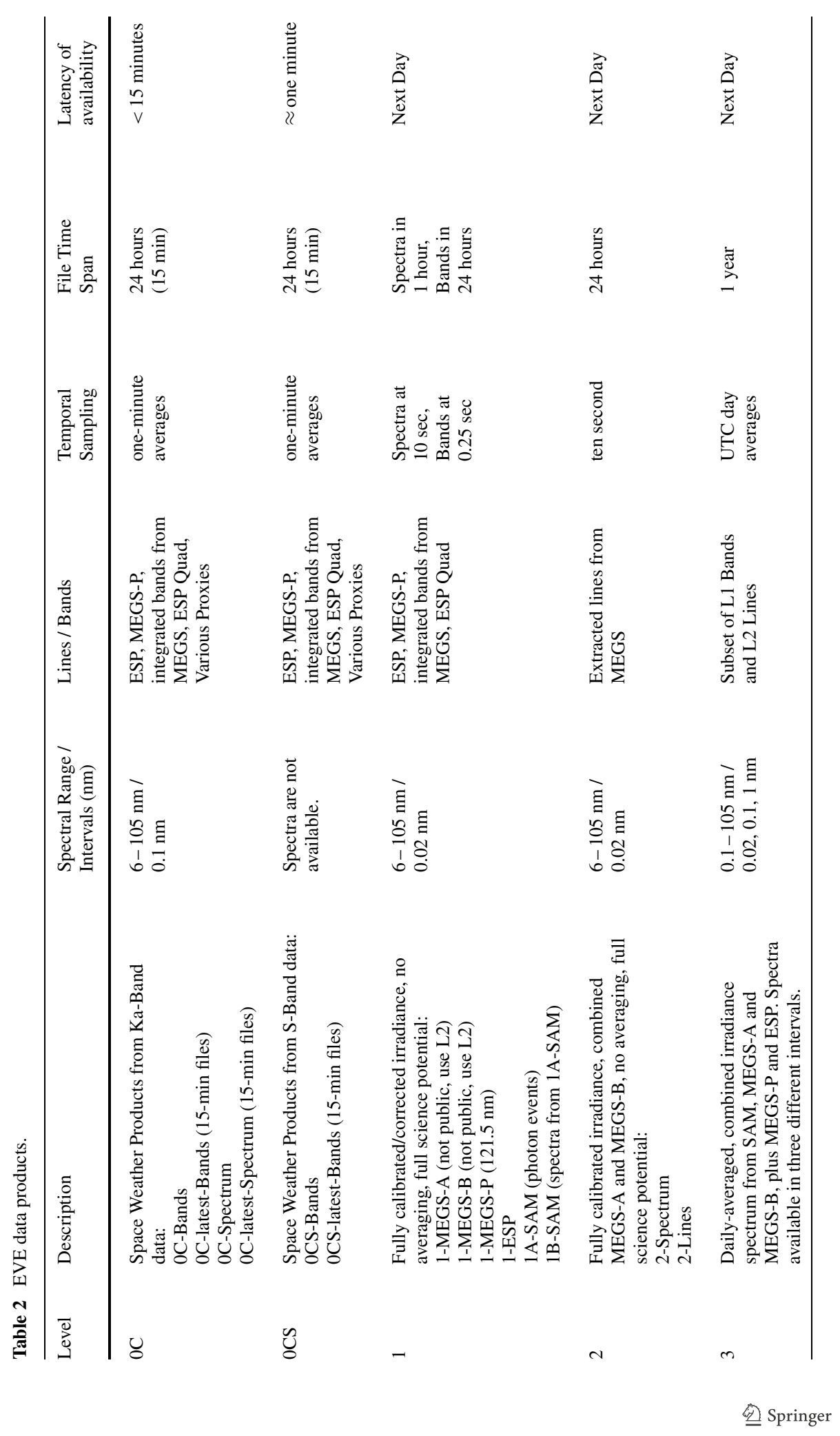


The space-weather products are all provided in ASCII files to support cross-platform compatibility and allow for human readability. The top of each file contains information (metadata) about the file contents and data formatting. Comment lines begin with the semicolon character. All space-weather products are generated on little-endian x86-based computers, so line endings in hexadecimal representation are 0x0a (LF). Windows-based computers must convert this to 0x0d (CR), 0x0a (LF). Each line represents a unique instant of time. We anticipate a latency of less than ten minutes for the routine, Ka-band spaceweather products. The ground system for SDO was not designed to accommodate real-time space-weather operations, so the high-rate data lag real-time by about three minutes. This is the time that it takes for data collected on the spacecraft to reach the science-processing operations center before any processing can begin.

The space-weather products are produced as rapidly as possible. Due to the desire to produce a faster product, the quality is lower and these products cannot meet the full science potential. These products are roughly calibrated using recent values for calibration parameters, and these products are not subject to accuracy requirements of the higher-level products, where latency is not a high priority.

Both Level 0C and 0CS space-weather products contain the ESP and MEGS-P broadband photometer measurements averaged to one-minute sampling. Each line of data corresponds to a one-minute average, and the file will be updated once per minute. These files will span one UT day. A smaller version of this file will also be made available which contains only the last 15 minutes. This reduced-size file will serve most real-time space-weather requests. A separate version of these EVE photometer bands is also available through the SDO Sband communication link, and these EVE Level 0CS products are expected to have less than one-minute latency.

The Level 0C space-weather product also contains one-minute averaged solar spectra at 0.1-nm sampling. Similar to the broadband file, this file will span one UT day with updates every minute. A reduced-size file containing only the most recent 15 minutes will also be produced to reduce download times for real-time space-weather needs.

\subsection{EVE Level 1}

The full science potential is achieved in the MEGS and ESP Level 1 science products. There is a Level 1 product for each of the EVE instruments: MEGS-A, MEGS-B, MEGS-SAM, MEGS-P, and ESP. The Level 1 products include spectra in $0.02 \mathrm{~nm}$ intervals and ten-second cadence and photometer bands with 0.25 -second cadence that has better than ten millisecond accuracy. For those who need composite spectra, where MEGS-A and MEGS- B spectra are combined, one can more conveniently use EVE's Level 2 and Level 3 data products that contain the full spectral coverage in a single product.

The Level 1 products contain every measurement collected with no temporal averaging, and the best calibration applied. After 14:00 UT each day the routine science processing can begin for the prior UT day. This provides time for the Mission Operations Center to ensure that the Level 0 files containing the ancillary science data packet information are complete. After this period the 48-node EVE computing cluster will begin Level 1 processing. The Level 1 processing is separated for each science component to allow parallel tasks to run simultaneously. MEGS-A slit 1 and slit 2 are stored together in one-hour files. MEGS-B is stored in separate one-hour duration files as well. However, MEGS-P is such a small amount of data that it is stored as a daily file, as is ESP. The remaining science instrument, SAM, is also divided into one-hour files. SAM will have a Level la event list (time, energy, location), from which the Level $1 \mathrm{~b}$ spectral product is derived. 


\subsection{EVE Level 2}

The Level 2 processing produces a combined set of merged spectra from 6 to $105 \mathrm{~nm}$ using the data from MEGS-A and MEGS-B, extracts solar emission features of special interest, and provides time-averaged photometer measurements to simplify comparisons with the spectra. This is done through a pair of files that will be produced: one file contains the spectra for one hour, and the other contains the lines and bands.

The spectrum files span one hour each. The individual ten-second spectra are sampled at a common, uniform wavelength spacing of $0.02 \mathrm{~nm}$. This provides five resolution samples per instrument resolution element $(0.1 \mathrm{~nm})$. The MEGS-A slit $1(6-16 \mathrm{~nm})$ and slit $2(16-$ $37 \mathrm{~nm})$ spectra are spliced together and then spliced together with MEGS-B $(37-105 \mathrm{~nm})$. The Level 2 spectrum files provide full instrument resolution at the full measurement cadence.

Table 3 lists the lines and bands planned for the EVE data products. The lines have been identified to include many plasma temperatures with good sampling between $20000 \mathrm{~K}$ and 10 MK. For example, this list includes the ionization states of Fe VIII through Fe XX. The extracted emission lines are total irradiances over the lines including the background. There are several broadbands including EVE's photometer measurements and several wavelength bands extracted from the MEGS spectra that simulate bands from other instruments, such as AIA, SOHO/Solar EUV Monitor (SEM) (Judge et al., 1998), and GOES/EUV Sensor (EUVS) (Viereck et al., 2007). For these other instrument bands, their normalized responsivity profiles are convolved with the MEGS spectrum so that the EVE equivalent bands can be more directly compared for calibration and validation purposes. Some additional bands may also be produced using the band definitions for the PROBA2/Large Yield Radiometer (LYRA) instrument (Hochedez et al., 2006), the EUVAC solar irradiance model (Richards, Fennelly, and Torr, 1994), JB2006 and JB2008 atmospheric models (Bowman et al., 2008a, 2008b), and TIM-GCM model solar input (Solomon and Qian, 2005).

\subsection{EVE Level 3}

The Level 3 products are merged files spanning one year of daily averages of the spectra. At this level, the daily-averaged SAM spectrum will also be provided from 0.1 to $6 \mathrm{~nm}$ with spectral resolution ranging from about $0.1 \mathrm{~nm}$ at $0.1 \mathrm{~nm}$ to about $1 \mathrm{~nm}$ at $6 \mathrm{~nm}$. The Level 3 product retains the high-resolution 0.02-nm sampling. An additional pair of products, integrated into $0.1-\mathrm{nm}$ and $1.0-\mathrm{nm}$ bins, will also be provided. The daily average is derived as the median value at each wavelength and thus normally represents the daily solar activity without flare contributions.

\subsection{EVE Data System}

The EVE data system was designed to be robust and fault tolerant to support near realtime operational product creation and distribution. The interface to the Data Distribution System (DDS) is comprised of twin (identical) redundant Solaris 10 (sparc) servers. This minimizes data interruptions for routine, planned system maintenance. Approximately oneminute duration data files received from the DDS are then passed to the processing cluster. A 48-node, high-availability, GNU-Linux cluster verifies the telemetry files, and performs the distributed data processing tasks. The head node runs a custom scheduler that assigns tasks to the cluster nodes. The tasks performed on each node are independent, so computer capacity may be increased by adding nodes. Furthermore, individual node failures have a 


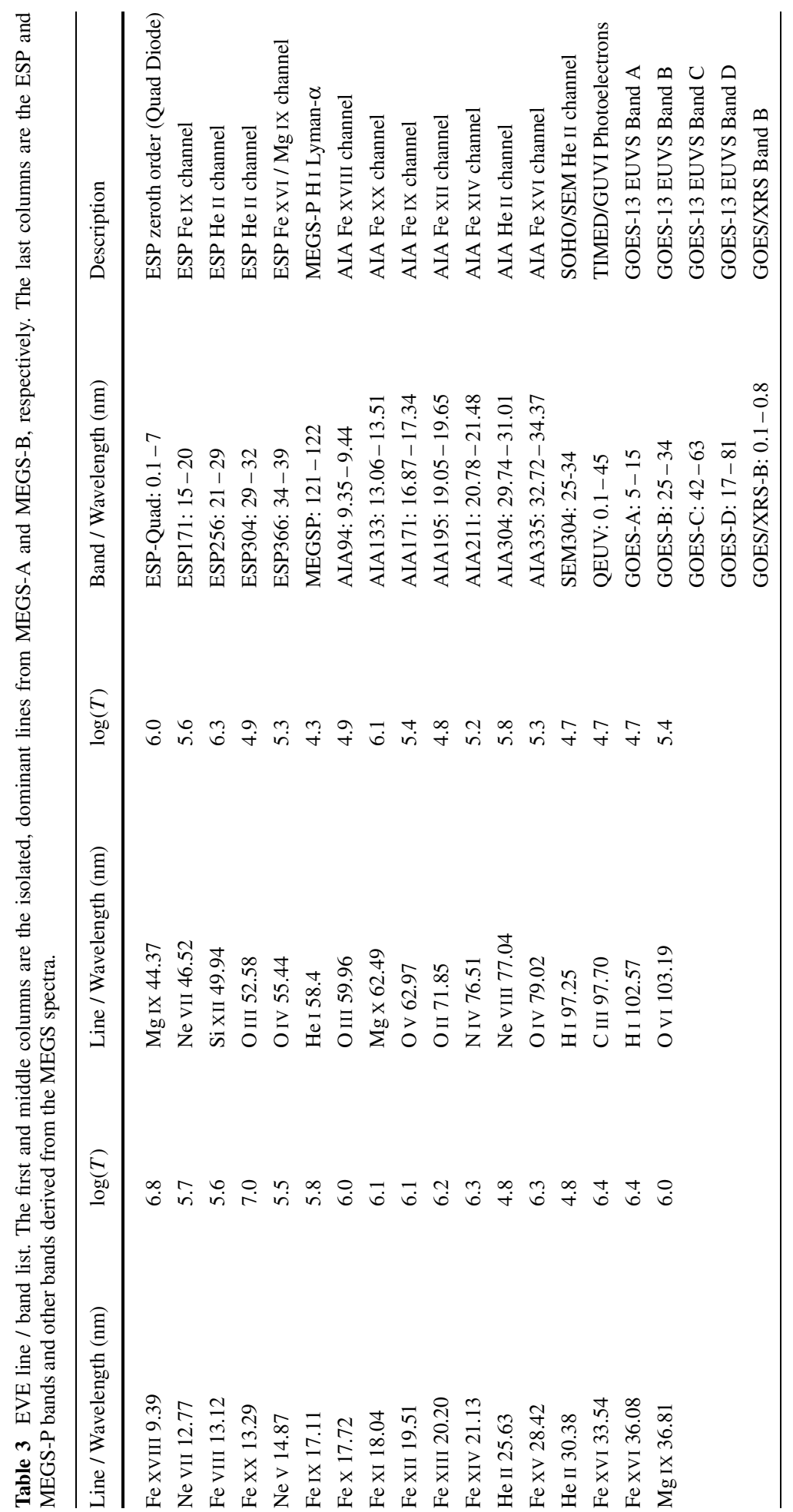


minimal impact on overall processing performance. Serialized processing is performed on each file as soon as it is verified to be complete and free of transmission errors. The Level 0B processing separates the packets from each science instrument, and in the case of MEGS-A and MEGS-B efficiently de-multiplexes and decodes each pixel (twos-complemented and shifted), and then assembles the CCD images into time-ordered integrations. The Level $0 \mathrm{~B}$ products are then used as input to generate space-weather products. The Level $0 \mathrm{~B}$ code is written in $\mathrm{C}$.

The largest source of data latency in the EVE space-weather processing is the approximately three-minute delay that was designed into the ground station at the White Sands Complex. This is needed since the DDS core system receives data from the antenna sites on a one-minute cadence, then separates the Virtual Channel Data Units (VCDUs) by instrument, stores data in a temporary 30-day disk storage repository, and performs statistics monitoring of the data and data files.

In addition to providing a repository of data products, the EVE Science Operations Center (SOC) is responsible for archiving the EVE data, and disseminating the processed data products. The EVE web site is http://lasp.colorado.edu/eve/.

\section{Solar Irradiance Models}

The EVE team plans to advance the understanding of EUV irradiance modulation by using magnetic features and their connections to surface magnetic-flux emergence. Physical processes will be developed, parameterized, and modeled, working toward the overall goal of a self-consistent, end-to-end formulation of EUV irradiance variations that arise from dynamo-driven magnetic fields. Although the primary focus is physics-based modeling, empirical approaches will also be employed to relate EUV irradiance variations to various proxies of solar activity that extend over long time periods, and into the future. These proxy models, once calibrated properly with the SDO measurements, are simpler to use for routine space-weather operations as long as the proxies have long-term stability (see, e.g., Lilensten et al., 2008).

A fundamental, new, and unique aspect of EVE-based modeling is physics-based simulations of EUV spectra in flares, for which detailed variations are not well known over the full EUV range. A preliminary attempt has been made to use differential-emission measures (DEMs) and fractional areas from TRACE, GOES, and SXT observations to model the increase in the EUV spectral irradiance during the Bastille Day 2000 flare (Meier et al., 2002). For operations since 2005, the SOLARFLARE model (Tobiska and Bouwer, 2005; Tobiska, 2007), which is part of SIP, uses GOES XRS 0.1 to $0.8 \mathrm{~nm}$ data, transformed into an effective temperature for the Mewe model, to create a 0.1-nm, one-minute flare spectrum from 0.1 to $30.0 \mathrm{~nm}$. Additionally, a few flares have been observed by TIMED/SEE and some empirical relationships have been developed for the FISM (Chamberlin, Woods, and Eparvier, 2008). According to these results, the EUV irradiance flare increase is comparable in magnitude to the solar-cycle increase, but has a remarkably different spectral shape. How realistic are these preliminary flare models? The new EVE and AIA data will enable the development of more accurate models of EUV flare variations. This effort will afford unique insights into the processes of flaring plasma, and significantly advance the capability for EUV irradiance specification on short time scales crucial for space-weather modeling.

EVE's solar modeling effort will also include quantitative studies of how the magnetic fields in the chromosphere and corona, which produce EUV irradiance sources, relate to surface flux emergence, transport, and rotation. This will be undertaken using the AIA and 
HMI images with a global scale focus. Relationships between coronal brightness and surface magnetic-field strengths will be investigated using potential-field extrapolation models. When combined with flux-transport models, these efforts will lead to new physics-based parameterizations between EUV irradiance and the solar dynamo that generates the surface flux. Furthermore, forecasting of the EUV irradiance is planned using helioseismic predictions of far-side active regions using HMI data.

These improved solar EUV irradiance models will be especially useful for space-weather operations after the SDO mission. The GOES/EUVS observations are expected to be operational during the SDO mission and to continue on past the SDO mission using a series of GOES satellites. The GOES/EUVS will only observe a few key EUV emissions (Viereck et al., 2007; Eparvier et al., 2009), so the EVE-established relationships of these GOES EUV bands to all other EUV wavelengths will be an important component for the future EUV irradiance-modeling effort.

\subsection{NRLEUV Model}

One approach to linking the global view of solar soft X-ray and EUV irradiance variability provided by EVE to the spatially-resolved AIA and HMI observations is the NRLEUV irradiance model (Warren, Mariska, and Lean, 2001; Warren, 2005). This model is currently based on four components of irradiance variability: coronal holes, quiet Sun, active network, and active regions. For the corona-hole, quiet-Sun, and active-region components, representative spectra derived from Skylab measurements are used to construct differential-emission-measure distributions. The DEM provides an empirical description of the density and temperature structure in the solar transition region and corona. Differentialemission measures for the active network component were determined by interpolating between the quiet-Sun and active-region distributions at transition region temperatures. These differential-emission-measure distributions were combined with databases of atomic physics parameters to compute the line intensity for any optically thin emission line (for, e.g., CHIANTI; Dere et al., 1997; Landi et al., 2006). Observed intensities are used in the model for optically thick emission lines. Once the line intensities are determined, the solar irradiance is computed by using full-disk solar images to estimate the contribution of each surface feature and simple limb-brightening curves to account for the increased path length near the limb. The original version of this model uses full-Sun coronal images from Yohkoh/SXT and chromospheric Ca II K line images from the Big Bear Solar Observatory. The NRLEUV formalism successfully reproduces much of the EUV irradiance during solar minimum conditions as well as the irradiance variability on solar-rotational time scales. The model tends to underestimate the solar-cycle variability for many wavelengths.

New observations from SDO will provide for significant improvements to the NRLEUV irradiance model. The broad temperature coverage, multiple channels, and absolute calibration of AIA will allow for accurate differential-emission measures to be computed at every position on the Sun at high cadence. These DEMs will be used to calculate the spectral radiance in each AIA pixel and eliminate both the need for crudely partitioning the Sun into broad intensity ranges and the use of limb-brightening curves at many wavelengths. The AIA 13.1-nm Fe XX/XXIII and 9.4-nm Fe XVIII channels will also provide spatially resolved images at very high temperatures. This will allow the NRLEUV model to be extended to flare temperatures and allow the model to capture very transient contributions to the solar irradiance. The NRLEUV model based on SDO will also incorporate the latest version of the CHIANTI atomic database (version 6), which contains more accurate atomic parameters. With these improvements the model will more accurately reproduce the irradiance variability co-temporally observed with EVE. The primary objective of the NRLEUV 
modeling effort will be to link changes in the solar soft X-ray and EUV irradiance with the evolution of the magnetic field over many different temporal and spatial scales. For example, the spatially resolved model will allow us to track the irradiance variability driven by the fragmentation and dispersal of active-region magnetic flux.

\subsection{FISM Model}

The Flare Irradiance Spectral Model (FISM) is an empirical model of the solar-irradiance spectrum from 0.1 to $190 \mathrm{~nm}$ at $1-\mathrm{nm}$ spectral resolution and on a one-minute time cadence (Chamberlin, Woods, and Eparvier, 2007, 2008). The goal of FISM is to provide accurate solar spectral irradiances over the aforementioned wavelength range as input for ionospheric and thermospheric models during times when no actual solar measurements are available. FISM is currently based on over seven years of measurements in the EUV $(0.1-119 \mathrm{~nm})$ from TIMED/SEE and the first five years of FUV observations from UARS/SOLSTICE $(119-190 \mathrm{~nm})$ for the daily components that model the solar-cycle and solar-rotational irradiance variations. TIMED/SEE also provides the FISM flare estimates for the entire wavelength range, but due to its $3 \%$ duty cycle there are only 30 flares (11 impulsive and 19 gradual phase observations) that make up the FISM basis data set.

EVE will contribute greatly in advancing the FISM model. Along with the improved accuracy of EVE over the existing SEE measurements, it will also provide more data from which to derive the FISM relationships of the measurements to the proxies. EVE will expand the base data set through Solar Cycle 24 improving the solar-cycle and solar-rotation components, but most importantly EVE will make available a much larger number of flare observations, as well as continuously observe the flares throughout their evolution due to its $100 \%$ duty cycle. EVE will also provide measurements at $0.1-\mathrm{nm}$ spectral resolution, and with the UARS/SOLSTICE and SORCE/SOLSTICE measurements this will allow for the entire FISM range to be enhanced to $0.1-\mathrm{nm}$ resolution once a statistically significant amount of data is collected.

\subsection{SIP Hybrid System of Data and Models}

Space Environment Technologies (SET) has developed the hybrid system Solar Irradiance Platform (SIP) as a superset of real-time data streams, reference spectra, models (empirical and physics-based) to provide irradiance product services that operationally mitigate space-weather adverse effects (Tobiska, 2008). For example, SET now operationally provides the JB2006, JB2008, F10.7, S10.7, M10.7, and Y10.7 solar proxies and indices that reduce the one- $\sigma$ uncertainty by up to $50 \%$ in atmosphere-density calculations for satellite orbit determination. SET operationally provides high time- and spectral-resolution solar irradiances that capture solar-flare effects on transionospheric communications for the Ionosphere Forecast Model (IFM) that is part of the Communication Alert and Prediction System (CAPS; http://spacewx.com, CAPS link under Products). These solar irradiance products have been developed and tested for $i$ ) daily time resolution for historical, nowcast, and intermediate-term forecast periods with one-day granularity, one-hour cadence, and onehour latency extending 4.5 months; ii) high time resolution for recent, nowcast, and shortterm forecast periods with three-hour granularity, one-hour cadence, and one-hour latency extending 96 hours; and iii) precision time resolution for recent, current epoch, and nearterm forecast periods with one-minute granularity, two-minute cadence, and five-minute latency extending to six hours. The SIP incorporates empirical and physics-based solar irradiance models such as SOLAR2000 (Tobiska, 2004, 2008; Tobiska and Bouwer, 2006; 
Tobiska et al., 2000) and SOLARFLARE (Tobiska and Bouwer, 2005; Tobiska, 2007) along with reference rocket measurements and real-time satellite data stream systems such as APEX for SOHO/SEM data as well as GOES/XRS and TIMED/SEE data.

The SOLARFLARE (SFLR) model has been coupled with SOLAR2000 in order to produce high time- and high spectral-resolution irradiances. SFLR is a mature model at the Technology Readiness Level (TRL) 9 that incorporates the Mewe model to produce 0.1-nm spectral lines based on solar coronal abundances. The Mewe model subroutine requires an input coronal electron temperature (in MK) to produce a spectrum. The GOES/XRS realtime data is collected from NOAA SWPC to predict coronal electron temperatures for use in the Mewe model, whose results are adjusted based on a calibration based on SORCE XPS measurements. The SFLR spectrum in the 0.05 to $30-\mathrm{nm}$ range, calculated at a two-minute cadence, is combined with the daily SOLAR2000 high-resolution spectrum longward of $30 \mathrm{~nm}$.

The solar-irradiance products from SIP will be improved with the new EVE measurements, notably important for the solar flare spectral variability and higher time cadence. In addition, solar reference spectra derived from EVE observations will be incorporated as products available through SIP.

\section{Earth's Atmospheric Models}

One of the four core objectives for the EVE program is to improve the understanding of how the solar EUV radiation affects the geospace environment, namely Earth's atmosphere above $50 \mathrm{~km}$. This research will entail incorporation of the solar EUV irradiance measurements into modeling the Earth's ionosphere and thermosphere and validation of the model results with atmospheric measurements during periods of interesting solar activity. Ultimately, the near real-time use of the EVE Level 0C space-weather product in some of these ionospheric and thermospheric models will represent an important milestone in the transition from research to operations for this aspect of the EVE program. These planned efforts are supported by EVE collaborators using the Time Dependent Ionospheric Model (TDIM; Schunk, 1988; Sojka, 1989), Ionosphere Forecast Model (IFM; Schunk and Sojka, 1996), and Global Assimilation of Ionospheric Measurements (GAIM; Schunk et al., 2004) at the Utah State University (USU), the Coupled Thermosphere Ionosphere Plasmasphere electrodynamics model (CTIPe) at NOAA (Fuller-Rowell et al., 1996, 2006), the NRLMSIS atmospheric model (Picone et al., 2002) and SAMI ionospheric model (Huba, Joyce, and Fedder, 2000) at NRL, and the JB2006 and JB2008 models (Bowman et al., 2008a, 2008b). We also encourage the involvement of the broader space-weather community in applying the EVE results towards advancing space-weather operations into more reliable forecasts.

\subsection{TDIM, IFM, and GAIM Models}

The Time Dependent Ionospheric Model (TDIM; Schunk, 1988; Sojka, 1989) uses a framework of physical processes to describe the global F-region ionosphere. This model includes time-dependent ion continuity and momentum equations, convection electric fields and particle precipitation, ion thermal conduction, and diffusion thermal heat flow in solving for the ion and electron densities and temperatures in the $\mathrm{F}$ region ionosphere. A derivative model is the Ionosphere Forecast Model (IFM; Schunk and Sojka, 1996), which is used as a physicsbased core in the Global Assimilation of Ionospheric Measurements (GAIM). The TDIM will be used to benchmark the ionospheric response to EVE solar irradiances. A similar 
study with TIMED/SEE solar EUV irradiances was completed early in the EVE development to help refine the EVE measurement requirements. The first EVE-based ionospheric studies will quantify the sensitivity of the ionosphere to the EUV spectrum, for example, determining the responses of the electron density and dynamics of the ionosphere to changes in the solar EUV spectrum. The model will then be used to determine which aspects of the solar spectrum are the most important and also to determine the temporal scales on which the ionosphere responds to solar EUV variability. This work will provide the foundation for EVE observations being used to drive other ionospheric models.

Models under development for operational space-weather forecasting, such as the Utah State University's GAIM model (Schunk et al., 2004), will rely heavily on accurate solar EUV measurements. GAIM is based on a background ionospheric model and then adjusts the electron densities using a Gauss - Markov Kalman filter of observations, such as from GPS ground receivers and Defense Meteorological Satellite Program (DMSP) satellites. A USU-USTAR initiative will run GAIM continuously with a temporal cadence of 15 minutes for routine space-weather operations as well as an evaluation test bed for GAIM models run in real-time at centers such as AFWA and NOAA. This 15-minute operational cadence of GAIM is a driving factor on providing EVE Level 0C spectra with a latency of less than 15 minutes.

\subsection{CTIPe Model}

The Coupled Thermosphere Ionosphere Plasmasphere electrodynamics (CTIPe) model at NOAA and CIRES (Fuller-Rowell et al., 1996; Millward et al., 1996; Codrescu et al., 2008) is a physical model that solves the dynamic differential equations for momentum, energy, and composition for the neutral and ionized medium, together with the electrodynamics at mid and low latitudes. Physical models such as CTIPe offer an advantage over climatologybased empirical models in that they provide a more detailed, time-dependent structure that is not available in a more statistical-type empirical solution. The physical model is also well suited for use in a data assimilation system in defining the current state and allowing for a more physically realistic evolution of the state (Fuller-Rowell et al., 2006).

CTIPe solves the equations for the thermosphere and ionosphere but requires external input of solar EUV radiation, solar-wind velocity, and interplanetary magnetic field to prescribe the magnetospheric input, and wave forcing from the lower atmosphere. The largest energy source on a day-to-day basis is the solar EUV irradiance so it is one of the key inputs for global modeling of the thermosphere and ionosphere system, as in CTIPe, to provide heating, ionization, and dissociation rates for the upper atmosphere. The accurate, high-time cadence EVE measurements of the solar EUV irradiance provide the opportunity to improve the description of solar forcing in the atmosphere rather than using the daily F10.7 to estimate the highly variable radiation at all EUV wavelengths. A real-time version of CTIPe is currently running at NOAA in a test operational mode to explore the potential application for space-weather forecasting. The EVE real-time observations will be used to drive CTIPe for real-time specification and forecasting of the ionosphere and thermosphere, and the EVE Level 0C space-weather products, once validated after the SDO launch, will be part of this effort.

\subsection{NRLMSIS Model}

NRLMSIS is an empirical, upper-atmosphere density specification model (Picone et al., 2002). The geospace community uses this model extensively, both for direct specification 
of neutral densities in operational activities and to provide the neutral-density component of other geospace models, for example the SAMI ionospheric model (Huba, Joyce, and Fedder, 2000). Given solar and geomagnetic inputs, the NRLMSIS estimates temperature, total mass density, and composition within the original MSIS framework (Hedin, 1991) at altitudes up to $1000 \mathrm{~km}$ for specified geographical location, time, and day of year. NRLMSIS utilizes daily F10.7, and time-centered 81-day running means, F10.7A, to simulate solar EUV radiation (adjusted from 1 AU to the appropriate Sun-Earth distance). The Ap index is a daily proxy for solar-wind-induced perturbations of the Earth's magnetic field. The total mass densities, temperatures, and composition shown in Figure 1 are global estimates from the NRLMSIS model, illustrating the large increases in average conditions from solar-cycle minimum and maximum (Figure 4).

The advent of extensive new databases of thermospheric densities derived from orbital drag, mass spectrometers, and UV remote sensing by the Global UV Imager (GUVI) on TIMED have afforded systematic, detailed validation and assessment studies of NRLMSIS (see, e.g., Emmert et al., 2006). Rarely is the model able to specify mass densities to better than $15 \%$, and more generally the uncertainties are larger, and often global in scale. A notable limitation of the NRLMSIS is recognized to be its use of F10.7 as an index for solar EUV irradiance variations. Whereas the F10.7 index mainly reflects changes in solar emissions from the corona, the strongest EUV emissions that dominate the Sun's energy input to the thermosphere and ionosphere originate in the solar chromospheres and transition region. This is motivating the development of improved density-specification models formulated using EUV irradiances (Emmert, Picone, and Meier, 2008). Lacking an adequate long-term EUV irradiance database over the past 30 years (the duration of the drag-derived density database), the new models utilize instead EUV irradiances calculated by an empirical model constructed from SEE observations in which each 1-nm irradiance bin is parameterized by a wavelength-dependent combination of the coronal F10.7 index and the chromospheric $\mathrm{Mg}$ index. Since the new density specification models are formulated for direct EUV irradiance inputs, they will ingest the EVE measurements directly, permitting rapid and effective incorporation of SDO observations to geospace applications.

\subsection{JB2006 and JB2008 Models}

The JB2006 empirical atmospheric density model (Bowman et al., 2008a) is developed using the CIRA72 (Jacchia, 1971) model as the basis for the diffusion equations. Solar indices based on on-orbit sensor data are used for the solar irradiances in the extreme and far ultraviolet wavelengths. Exospheric temperature and semiannual density equations are employed to represent the major thermospheric density variations. Temperature correction equations are also developed for diurnal and latitudinal effects, and finally density-correction factors are used for model corrections required at high altitude $(1500-4000 \mathrm{~km})$. The model is validated through comparisons of accurate daily density drag data previously computed for numerous satellites. For $400 \mathrm{~km}$ altitude, the standard deviation of $16 \%$ for the standard Jacchia model is reduced to $10 \%$ for the new JB2006 model for periods of low geomagnetic storm activity.

The JB2008 empirical atmospheric-density model (Bowman et al., 2008b) is developed as an improved revision to the JB2006 model. The solar indices for the solar irradiances have been extended to include X-ray and H I Lyman- $\alpha$ wavelengths. New exospheric temperature equations are developed to represent the thermospheric EUV and FUV heating. New semiannual density equations based on multiple 81-day average solar indices are used to represent the variations in the semiannual density cycle that result from EUV heating. 
Geomagnetic storm effects are modeled using the Dst index as the driver of global density changes. The model is validated through comparisons with accurate daily density drag data previously computed for numerous satellites in the altitude range of 175 to $1000 \mathrm{~km}$. Model comparisons have been computed for the JB2008, JB2006, Jacchia 1970, and NRLMSIS 2000 models. Accelerometer measurements from the CHAMP and GRACE satellites are also used to validate the new geomagnetic-storm equations.

\section{Summary}

SDO/EVE will measure solar EUV spectral irradiance with unprecedented spectral and temporal resolution and accuracy. These measurements will contribute to space-weather operations, and solar and atmospheric physics research, particularly solar flares and their effects on the geospace environment. EVE research will help validate and improve empirical and first-principle models of the solar irradiance variability and of the geospace environment. The EVE data will also extend the record of continuous solar EUV irradiance measurements from the SOHO/SEM and TIMED/SEE instruments, which started in 1996 and 2003 respectively. The EVE instrument suite completed its final pre-flight calibration in 2007 and is exceeding all of its instrument requirements. The SDO spacecraft integration and test (I\&T) activities have been completed at NASA's Goddard Space Flight Center (GSFC), and SDO currently awaits its launch in February 2010 (or later) into geosynchronous orbit for a nominal five-year mission.

Acknowledgements This research is supported by NASA contract NAS5-02140 to the University of Colorado. The authors gratefully acknowledge the many people who have contributed to the success of this new instrument throughout concept, design, fabrication, and testing. Special thanks to Vanessa George for her support in preparing this manuscript.

Open Access This article is distributed under the terms of the Creative Commons Attribution Noncommercial License which permits any noncommercial use, distribution, and reproduction in any medium, provided the original author(s) and source are credited.

\section{References}

Bertaux, J.-L., Quemerais, E., Lallement, R., Lamassoure, E., Schmidt, W., Kyrölä, E.: 2000, Geophys. Res. Lett. 27, 1331.

Bowman, B.R., Tobiska, W.K., Marcos, F.A., Valladares, C.: 2008a, J. Atmos. Solar Terr. Phys. 70, 774.

Bowman, B.R., Tobiska, W.K., Marcos, F.A., Huang, C.Y., Lin, C.S., Burke, W.J.: 2008b, In: AIAA 20086438, AIAA-AAS Astrodynamics Specialist Conference.

Chamberlin, P.C., Woods, T.N., Eparvier, F.G.: 2007, Space Weather J. 5, S07005. doi:10.1029/ 2007 SW000316.

Chamberlin, P.C., Hock, R.A., Crotser, D.A., Eparvier, F.G., Furst, M., Triplett, M.A., Woodraska, D., Woods, T.N.: 2007, SPIE Proc. 6689, 66890N. doi:10.1117/12.734116.

Chamberlin, P.C., Woods, T.N., Eparvier, F.G.: 2008, Space Weather J. 6, S05001. doi:10.1029/ 2007 SW000372.

Chamberlin, P.C., Woods, T.N., Crotser, D.A., Eparvier, F.G., Hock, R.A., Woodraska, D.L.: 2009, Geophys. Res. Lett. 36, L05102. doi:10.1029/2008GL037145.

Codrescu, M.V., Fuller-Rowell, T.J., Munteanu, V., Minter, C.F., Millward, G.H.: 2008, Space Weather J. 6, S09005. doi:10.1029/2007SW000364.

Crotser, D.A., Woods, T.N., Eparvier, F.G., Ucker, G., Kohnert, R., Berthiaume, G., Weitz, D.: 2004, SPIE Proc. 5563, 182.

Crotser, D.A., Woods, T.N., Eparvier, F.G., Triplett, M.A., Woodraska, D.L.: 2007, SPIE Proc. 6689, 66890M. doi:10.1117/12.732592. 
Dere, K.P., Landi, E., Mason, H.E., Monsignori Fossi, B.C., Young, P.R.: 1997, Astron. Astrophys. Suppl. 125, 149.

Didkovsky, L., Judge, D., Wieman, S., Woods, T., Chamberlin, P., Jones, A., Eparvier, F., Triplett, M., Woodraska, D., McMullin, D., Furst, M., Vest, R.: 2007, SPIE Proc. 6689, 66890P. doi:10.1117/ 12.732868.

Didkovsky, L., Judge, D., Wieman, S., Woods, T., Jones, A.: 2010, Solar Phys. doi:10.1007/s11207-0099485-8.

Emmert, J.T., Picone, J.M., Meier, R.R.: 2008, Geophys. Res. Lett. 35, L05101. doi:10.1029/2007GL032809.

Emmert, J.T., Meier, R.R., Picone, J.M., Lean, J.L., Christensen, A.B.: 2006, J. Geophys. Res. 111, A10S16. doi:10.1029/2005JA011495.

Eparvier, F.G., Woods, T.N., Crotser, D.A., Ucker, G.J., Kohnert, R.A., Jones, A., Judge, D.L., McMullin, D., Berthiaume, G.D.: 2004, SPIE Proc. 5660, 48.

Eparvier, F.G., Crotser, D., Jones, A.R., McClintock, W.E., Snow, M., Woods, T.N.: 2009, SPIE Proc. 7438, 743804.

Fontenla, J.M., Quemerais, E., Gonzalez Hernandez, I., Lindsey, C., Haberreiter, M.: 2009, Adv. Space Res. 44, 457.

Fuller-Rowell, T.J., Rees, D., Quegan, S., Moffett, R.J., Codrescu, M.V., Millward, G.H.: 1996, In: Schunk, R.W. (ed.) A Coupled Thermosphere-Ionosphere Model (CTIM), STEP Handbook, 217.

Fuller-Rowell, T.J., Codrescu, M.V., Minter, C.F., Strickland, D.: 2006, Adv. Space Res. 37, 401.

Garcia, H.: 1994, Solar Phys. 154, 275.

Hathaway, D.H., Wilson, R.M., Reichmann, E.J.: 1999, J. Geophys. Res. 104, 22375.

Hedin, A.: 1991, J. Geophys. Res. 96, 1159.

Hochedez, J.-F., Schmutz, W., Stockman, Y., Schühle, U., Benmoussa, A., Koller, S., Haenen, K., Berghmans, D., Defise, J.-M., Theissen, A., Delouille, V., et al.: 2006, Adv. Space Res. 37, 303.

Hock, R.A., Chamberlin, P.C., Woods, T.N., Crotser, D., Eparvier, F.G., Furst, M., Woodraska, D.L., Woods, E.C.: 2010, Solar Phys., in press.

Huba, J.D., Joyce, G., Fedder, J.A.: 2000, J. Geophys. Res. 105, 23035.

Jacchia, L.G.: 1971, Smithsonian Astrophys. Spec. Rep. 332, May.

Judge, D.L., McMullin, D.R., Ogawa, H.S., Hovestadt, D., Klecker, B., Hilchenbach, M., Mobius, E., Canfield, L.R., Vest, R.E., Watts, R.N., Tarrio, C., Kuhne, M., Wurz, P.: 1998, Solar Phys. 177, 161.

Knipp, D.J., Welliver, T., McHarg, M.G., Chun, F.K., Tobiska, W.K., Evans, D.: 2005, Adv. Space Res. 36, 2506.

Landi, E., Del Zanna, G., Young, P.R., Dere, K.P., Mason, H.E., Landini, M.: 2006, Astron. Astrophys. Suppl. 162, 261.

Lean, J.: 2005, Phys. Today 58, 32.

Lean, J.L., Picone, J.M., Emmert, J.T.: 2009, J. Geophys. Res. 114, A07301.

Lean, J.L., Warren, H.P., Mariska, J.T., Bishop, J.: 2003, J. Geophys. Res. 108, 1059.

Lilensten, J., Dudok de Wit, T., Kretzschmar, M., Amblard, P.O., Moussaoui, S., Aboudarham, J., Auchere, F.: 2008, Ann. Geophys. 26, 269.

Lindsey, C., Braun, D.C.: 2000, Science 287, 1799.

Marcos, F.A., Bowman, B.R., Sheehan, R.E.: 2006, In: AIAA 2006-6167, AIAA ASM.

Meier, R.R., Warren, H.P., Nicholas, A.C., Bishop, J., Huba, J.D., Drob, D.P., Lean, J., Picone, J.M., Mariska, J.T., Joyce, G., Judge, D.L., Thonnard, S.E., Dymond, K.F., Budzien, S.A.: 2002, Geophys. Res. Lett. 29, 99.

Millward, G.H., Moffett, R.J., Quegan, S., Fuller-Rowell, T.J.: 1996, In: Schunk, R.W. (ed.) Handbook of Ionospheric Models, STEP Report, 239.

National Space Weather Program: 2000, The Implementation Plan, 2nd edn., Office of the Federal Coordinator for Meteorology, FCM-P31-2000.

Pawlowski, D.J., Ridley, A.J.: 2008, J. Geophys. Res. 113, A10309.

Picone, J.M., Emmert, J.T., Lean, J.L.: 2005, J. Geophys. Res. 110, A03301. doi:10.1029/2004JA010585.

Picone, J.M., Hedin, A.E., Drob, D.P., Aikin, A.C.: 2002, J. Geophys. Res. 107(A12), 1468. doi:10.1029/ 2002JA009430.

Richards, P.C., Fennelly, J.A., Torr, D.G.: 1994, J. Geophys. Res. 99, 8981.

Schunk, R.W.: 1988, Ionospheric Modeling, Birkhäuser, Basel, 255.

Schunk, R.W., Sojka, J.J.: 1996, In: Schunk, R. (ed.) Solar-Terr. Energy Program: Handbook of Ionospheric Models, SCOSTEP, 153.

Schunk, R.W., Scherliess, L., Sojka, J.J., Thompson, D.C., Anderson, D.N., Codrescu, M., Minter, C., FullerRowell, T.J., Heelis, R.A., Hairston, M., Howe, B.M.: 2004, Radio Sci. 39, RS1S02.

Smithtro, C.G., Solomon, S.C.: 2008, J. Geophys. Res. 113, A08307.

Solomon, S.C., Qian, L.: 2005, J. Geophys. Res. 110, A10306.

Sojka, J.J.: 1989, Rev. Geophys. 27, 37. 
Tobiska, W.K.: 2004, Adv. Space Res. 34, 1736.

Tobiska, W.K.: 2007, AIAA 2007-0495.

Tobiska, W.K.: 2008, AIAA Proceedings, AIAA-2008-0453.

Tobiska, W.K., Bouwer, S.D.: 2005, In: Goodman, J.M. (ed.) 2005 Ionospheric Effects Symposium, MG Associates, Alexandria, 76.

Tobiska, W.K., Bouwer, S.D.: 2006, Adv. Space Res. 37, 347.

Tobiska, W.K., Woods, T., Eparvier, F., Viereck, R., Floyd, L., Bouwer, D., Rottman, G., White, O.R.: 2000, J. Atmos. Solar Terr. Phys. 62, 1233.

Viereck, R., Hanser, F., Wise, J., Guha, S., Jones, A., McMullin, D., Plunket, S., Strickland, D., Evans, S.: 2007, SPIE Proc. 6689, 66890K-10.

Wang, Y.-M., Sheeley, N.R. Jr.: 1991, Astrophys. J. 375, 761.

Wang, Y.-M., Lean, J., Sheeley, N.R. Jr.: 2000, Geophys. Res. Lett. 27, 505.

Wang, Y.-M., Sheeley, N.R. Jr., Lean, J.: 2000, Geophys. Res. Lett. 27, 621.

Wang, Y.-M., Lean, J.L., Sheeley, N.R. Jr.: 2005, Astrophys. J. 625, 522.

Warren, H.P.: 2005, Astrophys. J. Suppl. 157, 147. doi:10.1086/427171.

Warren, H.P., Mariska, J.T., Lean, J.: 2001, J. Geophys. Res. 106, 15745.

Westhoff, R.C., Rose, M.K., Gregory, J.A., Berthiaume, G.D., Seely, J.F., Woods, T.N., Ucker, G.: 2007, SPIE Proc. 6686, 668604. doi:10.1117/12.734371.

Woods, T.N., Rottman, G.J.: 2002, In: Mendillo, M., Nagy, A., Waite, J.H. Jr. (eds.) Comparative Aeronomy in the Solar System, Geophys. Monogr. Ser. 130, AGU, Washington, 221.

Woods, T., Acton, L.W., Bailey, S., Eparvier, F., Garcia, H., Judge, D., Lean, J., McMullin, D., Schmidtke, G., Solomon, S.C., Tobiska, W.K., Warren, H.P.: 2004, In: Pap, J., Fröhlich, C., Hudson, H., Kuhn, J., McCormack, J., North, G., Sprig, W., Wu, S.T. (eds.) Solar Variability and Its Effect on Climate, Geophys. Monogr. Ser. 141, AGU, Washington, 127.

Woods, T.N., Eparvier, F.G., Bailey, S.M., Chamberlin, P.C., Lean, J., Rottman, G.J., Solomon, S.C., Tobiska, W.K., Woodraska, D.L.: 2005, J. Geophys. Res. 110, A01312. doi:10.1029/2004JA010765.

Woodraska, D.L., Woods, T.N., Eparvier, F.G.: 2007, J. Spacecr. Rockets 44(6), 1204. doi:10.2514/1.28639. 